\title{
Giant in-particle field concentration and Fano resonances at light scattering by high-refractive-index particles
}

\author{
Michael I. Tribelsky* \\ Faculty of Physics, Lomonosov Moscow State University, Moscow 119991, Russia \\ and Moscow Technological University MIREA, Moscow 119454, Russia \\ Andrey E. Miroshnichenko ${ }^{\dagger}$ \\ Nonlinear Physics Centre, Australian National University, Canberra, Australian Capital Territory 2601, Australia
}

(Received 15 November 2015; published 31 May 2016)

\begin{abstract}
We present the results of a detailed analytical study of light scattering by a particle with high refractive index $m+i \kappa$ and low losses $(m \gg 1,0<\kappa \ll 1)$ based on the exact Mie solution. We show that there is a dramatic difference in the behavior of the electromagnetic field within the particle (inner problem) and outside it (outer problem). With an increase in $m$ at fixed values of the other parameters, the field within the particle asymptotically converges to a periodic function of $m$. The electric and magnetic type Mie resonances of different orders overlap substantially. It may lead to a giant concentration of the electromagnetic energy within the particle. At the same time, we demonstrate that the solution for the outer problem makes it possible to present each partial scattered wave as a sum of two partitions. One of them corresponds to the $m$-independent wave, scattered by a perfectly reflecting particle and plays the role of a background, while the other is associated with the excitation of a sharply $m$-dependent resonant Mie mode. The interference of the partitions brings about a typical asymmetric Fano profile. The profile is obtained from the exact Mie solution by means of identical transformations without any additional assumptions and/or fitting. It makes it possible to generalize rigorously the Fano theory to the case of finite dissipation. At an increase in $m$ the Fano resonances in the outer problem die out and the scattered field converges to the universal, $m$-independent profile. The behavior of the resonances at a fixed $m$ and varying particle size parameter $(x)$ is also discussed in detail. The similarities and differences of the two cases (fixed $x$, varying $m$ and fixed $m$, varying $x$ ) are disclosed. We also show that under certain very general conditions the scattering cross section of a large lossy sphere cannot be smaller than half its geometric cross section, while its absorption cross section cannot exceed three halves of the geometric one. Numerical estimates of most discussed effects for a gallium phosphide particle irradiated by the second harmonic of a Nd:YAG laser are presented as an example. In addition to purely academic interest, the obtained results may be employed to design new highly nonlinear heterogenic nanostructures and other metamaterials.
\end{abstract}

DOI: 10.1103/PhysRevA.93.053837

\section{INTRODUCTION}

Presently, the resonant light scattering by particles related to excitation of different eigenmodes attracts a great deal of attention of researchers all around the world [1-8]. In addition to purely academic interest, there is a broad spectrum of applications of the phenomenon in physics, chemistry, biology, medicine, data storage and processing, telecommunications, micro- and nanotechnologies, etc.; see, e.g., $[9,10]$. In particular, plenty of hopes were pinned on the resonant excitation of localized and/or bulk plasmons in metal nanoparticles [11]. Unfortunately, plasmonic resonances in such nanoparticles are usually accompanied by rather large dissipative losses, which in many cases diminish the advantages of the resonances. For this reason recently the frontier of the corresponding study has been shifted to light scattering by dielectric particles with high refractive index (HRI) $\hat{m}=m+i \kappa, m \gg 1$ and low losses $(\kappa \ll 1)$ [12]. In contrast to the plasmonic resonances, they exhibit the high- $Q$ Mie resonances of both electric and magnetic types [13-15], which bring more opportunities for wider applications in

\footnotetext{
*tribelsky@mirea.ru

${ }^{\dagger}$ Corresponding author: andrey.miroshnichenko@anu.edu.au
}

sensing, spontaneous emission enhancement, unidirectional scattering, etc.

Despite the fact that the exact Mie solution, describing light scattering by a sphere with an arbitrary size and material properties, has been known for more than 100 years and the case of a sphere with HRI has been repeatedly discussed in textbooks and monographs (see, e.g., [16,17]), some important peculiarities of this problem have not been disclosed yet. Meanwhile, the quantitative feature of HRI brings about qualitatively new, unusual effects and paradoxes, which merely do not exist at moderate values of the refractive index $[13,18,19]$.

The aim of this paper is to fill the gap in the understanding of the light scattering by a HRI homogeneous particle. Specifically, in contrast to the common belief that in this case the field within the particle should be weak, we show that the scattering may be accompanied by a giant concentration of the electromagnetic field within the particle. We also reveal the nature of the Fano resonances exhibited by partial scattered waves and rigorously extend their description to a scatter with dissipation. Though in what follows just the Mie solution for a sphere is discussed, the results obtained may be readily generalized to a cylinder and any other analytically tractable case. The general discussion is illustrated by calculations of the scattering of light with the wavelength $532 \mathrm{~nm}$ by 
a nanosphere made of gallium phosphate. These estimates are typical for other widely used semiconductors ( $\mathrm{Si}, \mathrm{Ge}$, GaAs, etc.). We believe that in addition to the shedding a light on the mentioned fundamental problem, our results may be important for numerous applications, especially in the design of new generations of highly nonlinear heterogenic optical nanostructures and other metamaterials [20]. The paper presented here is a revised and extended version of Ref. [21].

The paper is arranged as follows. In Sec. II the problem formulation is presented. In Sec. III we consider general properties of the Mie solution at the limit of high, purely real $m$. In Sec. IV the line shapes and linewidths of partial resonances for the scattered field and the field concentrated within the particle are inspected. In Sec. V the generic nature of the Fano resonances for partial modes of the scattered field is disclosed, and the explicit expressions for the parameters of the Fano profile are obtained from first principles by means of identical transformations of the exact Mie solution. In Sec. VI we show that in the nondissipative limit the entire set of the infinite number of the cascades of the resonances possesses a certain scaling and may be reduced to a universal set of lines by simple scale transformations. In Sec. VII, effects of finite dissipation are inspected. In Sec. VIII the resonances at a fixed $m$ and varying size parameter are discussed and the manifestation of the resonances in a particle made of gallium phosphide is presented as an example. In the Conclusions section a brief summary of the obtained results is presented. In the Appendix certain cumbersome but important calculations are performed.

\section{THE PROBLEM FORMULATION}

It is well known that at the limit $m \rightarrow \infty$ a small (relative to the incident light wavelength) dielectric sphere scatters light as a perfectly reflecting one "into which neither the electric, nor the magnetic field penetrates" [16]. Then it may be concluded that the electromagnetic field within the scattering particle should vanish at $m \rightarrow \infty$. Seemingly, the conclusion is supported by the argument that a HRI implies a high polarizability of the sphere. Then, from the point of view of the polarizability by an external electric field at $m \rightarrow \infty$, a dielectric sphere becomes equivalent to a perfectly conducting one [16], and the electric field induced within the particle owing to its polarization by the incident light should compensate the field inducing the polarization. That is to say, the field within the particle should vanish.

In fact, the question is much more subtle, and the actual situation is far from this simple picture. The point is that the wavelength inside the particle vanishes at $m \rightarrow \infty$. Then, at large-enough $m$ the wavelength within the particle becomes smaller than the particle size, no matter how small geometrically the particle is. In this case the incident wave may resonantly excite in the particle the Mie electromagnetic eigenmodes. Moreover, an unlimited growth in $m$ results in infinite cascades of these resonances; see below. The interference of the resonant eigenmodes with the incident wave and, what is most important, with each other gives rise to dramatic changes in the aforementioned simple scattering process. To reveal these changes is the goal of our study. To this end the full Mie theory is employed.
We show that at $m \rightarrow \infty$ the scattered field for the outer problem does converge to the one for the sphere made of a perfect electric conductor (PEC), no matter whether the sphere is small or large. In contrast, though it sounds paradoxical, the field within the particle does not have any limit at all. Such a difference between the outer and inner problems is related to the different line shapes of the Mie resonances in the two problems. For the former an increase in $m$ makes the resonances less pronounced. For the latter in the nondissipative limit the amplitude of the resonances increases with an increase in $m$. In the case of a finite dissipation rate (regardless how small it is), the growth of the amplitudes eventually saturates and the resonance lines become periodic functions of $m$. In both cases (with and without dissipation) the field within the particle does not tend to any fixed limit at $m \rightarrow \infty$.

It is important to stress that the resonance lines of different orders and different origin (i.e., electric and magnetic) may overlap substantially. Note that overlaps of resonances have been extensively studied in optics. It is well known that they may result in many interesting effects; see, e.g., [22,23]. Here we discuss the overlap in connection with the problem in question.

The mentioned peculiarities of the inner field in the vicinity of the resonances may result in a giant concentration of the electromagnetic energy inside the particle. At realistic values of the refractive index, the proper selection of the particle radius and/or the wavelength of the incident radiation the field inside the particle may exceed the one in the incident wave by several orders of magnitude. Such a huge field may give rise to numerous nonlinear effects. For this reason the discussed results may appear extremely important in the design and fabrication of highly nonlinear nanostructures.

Regarding the outer problem, it is known that the scattering coefficients in the one have the well-pronounced asymmetric Fano resonance profiles. Recent publications of Rybin et al. (Refs. [24-26]) should be mentioned in this context. Based on the analysis of the exact Lorenz-Mie solution for a cylinder, the authors of these publications have revealed that the resonant Mie scattering can be presented through infinite cascades of the Fano resonances caused by the interference between the narrow-line resonant Mie scattering and the nonresonant (background) scattering from the object. The analytical expressions for both the partitions have been obtained through the Maxwell boundary conditions. The numerical fit of the line shape, resulting from the exact solution in the vicinity of the resonances, to the conventional Fano profile [27] has allowed the authors of Refs. [24-26] to obtain the dependence of the Fano asymmetry parameter $q$ [27] on the ratio of the radius of the cylinder $R$ to the wavelength of the incident light $x=2 \pi R / \lambda$ (size parameter) in rather a broad range of its variations. They also have shown that in the inspected cases $q(x) \sim \cot x$. This dependence agrees with their previous results for disordered photonic crystals [28], as well as with the general expression for $q$ in terms of the phase shift of the background partition [29].

Nonetheless, despite the observation of the cascades of the Fano resonances at light scattering by a particle, these authors have not disclosed the physical nature of the background partition. Regarding the results obtained by the numerical fit, the great advantage of this procedure is the possibility to fit any 
curve with any set of the basic functions. However, precisely because of that, based on the fitting solely, one can never answer the question whether the studied profile is the Fano profile indeed, or it is just fitted to that profile. It also remains unclear how far beyond the inspected numerical domain the obtained results could be extended; e.g., what happens with the modes with the multipolarity higher than that examined by the authors, etc.

For this reason, a self-consistent analytical examination of the problem, connecting the parameters of the Fano profiles with the fundamental parameters of the light scattering $x$ and $m$ would be highly desirable. Such a study is produced in the present paper. Specifically, we show that the Fano profile may be obtained by identical transformations of the exact Mie solution. As a result, the exact analytical expressions for the parameters of the profile follow from this transformation automatically. Regarding the background partition, we reveal that it is just the corresponding partial wave scattered by a PEC sphere with the same $x$. Another advantage of our approach is the rigorous extension of the concept of the Fano resonances to dissipative systems.

We should emphasize that the resonances discussed in the present paper have nothing to do with the well-known whispering gallery modes. These modes are associated with the waves propagating along the surface of the particle, confined there by "continuous total internal reflection" [30] and cannot be excited by a plane incident wave. Closest to the topic of our study is another well-known phenomenon: the ripple structure in the spectrum at light scattering by droplets with $m \approx 1.5$ (see, e.g., [17,31,32]), with accidental ultrasharp resonances [33] and related problems. The resonances we discuss and the ripple structure have the same nature. However, the large value of the refractive index makes the manifestation of our resonances quite different from what is known for the ripple structure. In addition, the specific characteristics of the resonances examining in our paper usually are not discussed in connection with the ripple structure.

Note also the very close effects discussed in Ref. [34] for a magnetic particle. However, up to now materials with large magnetic permeability at optical frequencies remain hypothetical objects, while the ones with HRI may be easily found among the common semiconductors; see below.

It is necessary to stress in this context that all the effects discussed in the present paper occur to optically thick particles, i.e., $R$ should be about or larger than $\lambda / m$, where $\lambda$ is the wavelength of the incident light. Regarding $R$, from here and in what follows it designates the radius of the sphere. The important peculiarity of the HRI particles is that an optically thick particle simultaneously may be geometrically small with $R \ll \lambda$; cf. [18].

Last but not least, the only thing we do below is a detailed analysis of the well-known Mie solution at the range of high refractive index of the scattering sphere. It would have been nothing but a mathematical exercise if it did not reveal new unusual features of the phenomenon. It did. Let us proceed discussing these features in detail.

\section{HIGH REFRACTIVE INDEX LIMIT}

The subsequent analysis is based upon the exact Mie solution describing light scattering by a spatially homogeneous sphere with an arbitrary radius $R$ and a given permittivity $\varepsilon$. The case of a cylinder and core-shell structures may be inspected in the same manner.

Since we are interested in the optical properties of a particle with low dissipation, we first focus on a discussion of the nondissipative limit, i.e., a purely real positive refractive index $m \equiv \sqrt{\varepsilon}$. The general case of a complex refractive index along with the applicability condition for the nondissipative limit will be produced later on.

According to the Mie solution, the scattered field is presented as an infinite series of partial multipolar contributions (dipolar, quadrupolar, etc.) of the two types: the so-called electric and magnetic modes. The modes differ in the radial components of fields $\mathbf{E}$ and $\mathbf{H}$. For the electric modes $H_{r}=0$; for magnetic modes $E_{r}=0$. For this reason the former and the latter are often called transverse magnetic and transverse electric waves, respectively [35]. For the sake of brevity in what follows only the electric modes are discussed in detail. The behaviors of the magnetic modes usually are alike. Therefore, the corresponding discussion of these modes is rather brief.

The key quantities of the Mie solution are the properly normalized complex amplitudes of the field components. Two of them, $a_{n}$ and $b_{n}$, are related to the scattered field outside the particle. Two others, $c_{n}$ and $d_{n}$, describe the internal field within the particle. These coefficients should satisfy the boundary conditions, following from the continuity of the tangential components of the electric and magnetic fields on the surface of the particle. The conditions are split into two independent pairs for the electric $\left(a_{n}, d_{n}\right)$ and magnetic $\left(b_{n}, c_{n}\right)$ modes, respectively [36],

$$
\begin{aligned}
\xi_{n}(x) a_{n}+\psi_{n}(m x) d_{n} & =\psi_{n}(x), \\
m \xi_{n}^{\prime}(x) a_{n}+\psi_{n}^{\prime}(m x) d_{n} & =m \psi_{n}^{\prime}(x), \\
m \xi_{n}(x) b_{n}+\psi_{n}(m x) c_{n} & =m \psi_{n}(x), \\
\xi_{n}^{\prime}(x) b_{n}+\psi_{n}^{\prime}(m x) c_{n} & =\psi_{n}^{\prime}(x) .
\end{aligned}
$$

Here $x=2 \pi R / \lambda$ is the size parameter for the sphere; $\psi_{n}(z)$, $\xi_{n}(z)=\psi_{n}(z)-i \chi_{n}(z), \psi_{n}(z)=z j_{n}(z)$, and $\chi_{n}(z)=-z y_{n}(z)$ are the Riccati-Bessel functions; $j_{n}(z)$ and $y_{n}(x)$ stand for the spherical Bessel functions $[36] ;{ }^{\prime} \equiv \partial / \partial z$ designates derivative relative to the entire argument. Integer $n$ indicates the multipolarity of the mode, so that $n=1,2, \ldots$ correspond to the dipole mode, quadrupole mode, etc. It should be stressed that functions $\psi_{n}(z)$ and $\chi_{n}(z)$ are real for real $z$.

Solving Eqs. (3.1) and (3.2) with respect to $a_{n}$ and $d_{n}$, we obtain [36]

$$
\begin{aligned}
a_{n} & =\frac{m \psi_{n}(m x) \psi_{n}^{\prime}(x)-\psi_{n}(x) \psi_{n}^{\prime}(m x)}{m \psi_{n}(m x) \xi_{n}^{\prime}(x)-\xi_{n}(x) \psi_{n}^{\prime}(m x)}, \\
d_{n} & =\frac{i m}{m \psi_{n}(m x) \xi_{n}^{\prime}(x)-\xi_{n}(x) \psi_{n}^{\prime}(m x)},
\end{aligned}
$$

where the identity

$$
\psi_{n}(x) \xi_{n}^{\prime}(x)-\psi_{n}^{\prime}(x) \xi_{n}(x) \equiv i,
$$


following from the expression for the Wronskian of the Riccati-Bessel functions $\left(\chi_{n} \psi_{n}^{\prime}-\psi_{n} \chi_{n}^{\prime} \equiv 1\right)$ [37], has been employed.

The corresponding treatment of the magnetic modes yields

$$
\begin{aligned}
b_{n} & =\frac{m \psi_{n}(x) \psi_{n}^{\prime}(m x)-\psi_{n}(m x) \psi_{n}^{\prime}(x)}{m \xi_{n}(x) \psi_{n}^{\prime}(m x)-\psi_{n}(m x) \xi_{n}^{\prime}(x)}, \\
c_{n} & =-\frac{i m}{m \xi_{n}(x) \psi_{n}^{\prime}(m x)-\psi_{n}(m x) \xi_{n}^{\prime}(x)} .
\end{aligned}
$$

Note that, according to Eqs. (3.6), (3.7), and (3.9), $d_{n} \equiv c_{n} \equiv 1$ at $m=1$, i.e., when the optical properties of the particle are identical to those of the embedding medium. Thus, $\left|d_{n}\right|$ and $\left|c_{n}\right|$ may be regarded as the enhancement parameters for the field within the particle.

Equations (3.5), (3.6), (3.8), and (3.9) may be written in the equivalent, identical form,

$$
\begin{aligned}
& a_{n}=\frac{F_{n}^{(a)}}{F_{n}^{(a)}+i G_{n}^{(a)}}, \quad d_{n}=\frac{i m}{F_{n}^{(a)}+i G_{n}^{(a)}}, \\
& b_{n}=\frac{F_{n}^{(b)}}{F_{n}^{(b)}+i G_{n}^{(b)}}, \quad c_{n}=-\frac{i m}{F_{n}^{(b)}+i G_{n}^{(b)}},
\end{aligned}
$$

with

$$
\begin{gathered}
F_{n}^{(a)}=m \psi_{n}^{\prime}(x) \psi_{n}(m x)-\psi_{n}(x) \psi_{n}^{\prime}(m x), \\
G_{n}^{(a)}=-m \chi_{n}^{\prime}(x) \psi_{n}(m x)+\chi_{n}(x) \psi_{n}^{\prime}(m x), \\
F_{n}^{(b)}=m \psi_{n}(x) \psi_{n}^{\prime}(m x)-\psi_{n}(m x) \psi_{n}^{\prime}(x), \\
G_{n}^{(b)}=-m \chi_{n}(x) \psi_{n}^{\prime}(m x)+\chi_{n}^{\prime}(x) \psi_{n}(m x) .
\end{gathered}
$$

Let us focus on the electric modes. At the limit of large refractive index we can keep just the first terms in Eqs. (3.12) and (3.13):

$$
\begin{gathered}
F_{n}^{(a)} \underset{m \gg 1}{\longrightarrow} m \psi_{n}^{\prime}(x) \psi_{n}(m x), \\
G_{n}^{(a)} \underset{m \gg 1}{\longrightarrow}-m \chi_{n}^{\prime}(x) \psi_{n}(m x) .
\end{gathered}
$$

Equations (3.16) and (3.17) lead to two important conclusions. First, the internal coefficients $d_{n}$ in this limit converge to

$$
d_{n} \underset{m \gg 1}{\longrightarrow} d_{n}^{(\lim )}=\frac{i}{\psi_{n}(m x) \xi_{n}^{\prime}(x)},
$$

i.e., remain $m$ dependent.

Second, in contrast to $d_{n}$, the partial scattering coefficients $a_{n}$ converge to the $m$-independent form, valid for a PEC sphere,

$$
a_{n} \underset{m \gg 1}{\longrightarrow} a_{n}^{(\mathrm{PEC})}=\frac{F_{n}^{(a, \mathrm{PEC})}}{F_{n}^{(a, \mathrm{PEC})}+i G_{n}^{(a, \mathrm{PEC})}}=\frac{\psi_{n}^{\prime}(x)}{\xi_{n}^{\prime}(x)},
$$

with

$$
F_{n}^{(a, \mathrm{PEC})}=\psi_{n}^{\prime}(x), \quad G_{n}^{(a, \mathrm{PEC})}=-\chi_{n}^{\prime}(x) .
$$

It is relevant to mention that while expressions (3.18) and (3.19) satisfy boundary condition (3.1) identically, boundary condition (3.2) for these expressions is satisfied only asymptotically at $m \rightarrow \infty$.

Expression (3.19) can be also obtained from the boundary condition [Eq. (3.2)] if we suppose there that $d_{n}=0$, as it should for a PEC, or by direct solution of the light-scattering problem for PEC.

We have to emphasize here that the problem of light scattering by a PEC is a bit tricky. The point is that the field inside the PEC is zero identically. Then, instead of the four independent scattering coefficients - the two for the scattered field outside the particle and the two for the field within the particle - only the former two remain. The reduction of the independent constants of integration of the Maxwell equations requires the corresponding reduction of the boundary conditions. For PEC the boundary conditions stipulate vanishing of the tangential component of the electric field at the surface of the sphere [38]. Eventually, they result in the expressions which may be obtained from Eqs. (3.1)-(3.4) if we drop there Eqs. (3.1), (3.4) and set $d_{n}=c_{n}=0$ in remaining Eqs. (3.2) and (3.3).

Another type of perfect reflection corresponds to the socalled perfect magnetic conductor (PMC). In this case the boundary conditions require the vanishing of the tangential components of the magnetic field [38]. For PMC the corresponding reduction of Eqs. (3.1)-(3.4) keeps Eqs. (3.1) and (3.4) supplemented by the same conditions $d_{n}=c_{n}=0$, while Eqs. (3.2) and (3.3) should be removed. The case of PMC is achieved when $\mu \rightarrow \infty$, where $\mu$ is the particle magnetic permeability. Since at the optical frequencies $\mu=1$ the case of PMC has nothing to do with the problem in question and is not discussed here.

It is interesting to note also that, while limit Eqs. (3.19) and (3.20) corresponds to a perfect electric conductor, for the problem under consideration a sphere is supposed to be made of a perfect insulator with zero conductivity $(\operatorname{Re} \varepsilon \gg 1$, $\operatorname{Im} \varepsilon=0$ ). The coincidence of the two limits occurs owing to the fact that, despite the vanishing of the conduction current in our case, the displacement current plays its role. If the field $\mathbf{E}$ inside the particle does not vanish, the displacement current diverges at $\operatorname{Re} \varepsilon \rightarrow \infty$. To avoid the divergence the electric field inside the particle must be set equal to zero.

It should be stressed that limit (3.19) is not valid in the vicinity of the points, where $\psi_{n}(m x)=0$ owing to departures of $F_{n}^{(a)}$ and $G_{n}^{(a)}$ from asymptotic expressions (3.16) and (3.17) in these regions, no matter how large $m$ is. On the other hand, exactly at these points $\left|d_{n}^{(\text {lim) }}\right| \rightarrow \infty$; see Eq. (3.18). Regarding $\left|d_{n}\right|$, they undergo a sharp growth but remain finite due to the mentioned properties of $F_{n}^{(a)}$ and $G_{n}^{(a)}$; see below for more details. Such a behavior of $d_{n}$ allows us to conclude that in a HRI dielectric sphere at large-enough $m$ in the vicinity of the points defined by the condition

$$
\psi_{n}(m x)=0
$$

the resonant excitation of the electric eigenmodes occurs.

This resonance condition can be further simplified in the socalled Fraunhofer regime, when the Riccati-Bessel functions are reduced to simple trigonometrical ones. Specifically, in this case

$$
\psi_{n}(z) \cong \sin \left(z-\frac{n \pi}{2}\right)
$$


so that expression $\psi_{n}(m x)=0$ reads

$$
\sin \left(m x-\frac{n \pi}{2}\right)=0 .
$$

Strictly speaking, the applicability condition for the Fraunhofer regime is $z \gg n^{2}$. However, the Fraunhofer asymptotics still provide an acceptable approximation of $\psi_{n}(z)$ and $\chi_{n}(z)$ for $z$ as small as $z \approx 2 n$ [39]. Therefore, in the present study the range of validity of the Fraunhofer regime, as a rule, is adopted as $z \geqslant 2 n$.

An analogous treatment of the magnetic coefficients $b_{n}$ (for the outer problem) and $c_{n}$ (for inner) brings about the expressions

$$
\begin{gathered}
F_{n}^{(b)} \underset{m \gg 1}{\longrightarrow} m \psi_{n}(x) \psi_{n}^{\prime}(m x), \\
G_{n}^{(b)} \underset{m \gg 1}{\longrightarrow}-m \chi_{n}(x) \psi_{n}^{\prime}(m x), \\
b_{n} \underset{m \gg 1}{\longrightarrow} b_{n}^{(\mathrm{PEC})}=\frac{F_{n}^{(b, \mathrm{PEC})}}{F_{n}^{(b, \mathrm{PMS})}+i G_{n}^{(b, \mathrm{PEC})}}=\frac{\psi_{n}(x)}{\xi_{n}(x)}, \\
F_{n}^{(b, \mathrm{PEC})}=\psi_{n}(x), \quad G_{n}^{(b, \mathrm{PEC})}=-\chi_{n}(x), \\
c_{n} \underset{m \gg 1}{\longrightarrow} c_{n}^{(\mathrm{lim})}=-\frac{i}{\psi_{n}^{\prime}(m x) \xi_{n}(x)},
\end{gathered}
$$

with the condition for the magnetic modes resonances

$$
\psi_{n}^{\prime}(m x)=0
$$

leading in the Fraunhofer regime to the equation

$$
\psi_{n}^{\prime}(m x) \cong \cos \left(m x-\frac{n \pi}{2}\right)=0 .
$$

It is interesting to discuss Eqs. (3.21) and (3.29) from the viewpoint of the boundary conditions imposed on fields $\mathbf{E}$ and $\mathbf{H}$ of a resonant mode on the surface of the sphere from inside, i.e., within the particle. To avoid misunderstanding, we have to remind the reader that boundary conditions (3.1)-(3.4) are derived from the primary boundary conditions which stipulate continuity of the tangential components of $\mathbf{E}$ and $\mathbf{H}$ on the surface of the sphere. These primary conditions are identical to Eqs. (3.1)-(3.4), but look different [36]. We do not present them here.

Inspection of the primary boundary conditions for the fields shows that in the case of the electric mode resonance [Eq. (3.21)] the tangential components of field $\mathbf{H}$ for the resonant electric mode vanish on the surface of the sphere. Since the radial components of $\mathbf{H}$ for any electric mode by definition is zero, it means that in this case the entire field $\mathbf{H}$ for the resonant electric mode vanishes on the surface. Regarding the electric field of this mode, under condition (3.21) its radial component vanishes, so the field becomes tangential to the surface.

In the case of the magnetic mode resonance [Eq. (3.29)] only tangential components of field $\mathbf{H}$ for the resonant magnetic mode vanish. Other components of $\mathbf{E}$ and $\mathbf{H}$ do not exhibit any peculiarities at this type of resonance.

An unexpected conclusion, following from these features is that at both types of the resonances for the field within the sphere its surface acts as a PMC mirror, forcing the tangential component of $\mathbf{H}$ to vanish on the boundary.
Resonance conditions (3.23) and (3.30) may be rewritten in a unified form:

$$
\begin{gathered}
m_{n, p}^{(\mathrm{res}, E)} \cong(n+2 p) \frac{\pi}{2 x}, \\
m_{n, p}^{(\mathrm{res}, H)} \cong(n+2 p+1) \frac{\pi}{2 x},
\end{gathered}
$$

where $p$ is a non-negative integer number. The meaning of resonance conditions (3.31) and (3.32) becomes absolutely clear if we recall that $x=2 \pi R / \lambda$ and $\lambda / m$ is the wavelength inside the scattering particle. Then, Eqs. (3.31) and (3.32) may be presented as

$$
\begin{aligned}
& \frac{\lambda}{m_{n, p}^{(\mathrm{res}, E)}}\left(\frac{n}{2}+p\right) \cong 2 R, \\
& \frac{\lambda}{m_{n, p}^{(\mathrm{res}, H)}}\left(\frac{n+1}{2}+p\right) \cong 2 R
\end{aligned}
$$

i.e., the resonances occur when an integer number of the halfwaves equals the diameter of the sphere (cf. [14]).

These resonance conditions lead to a number of interesting conclusions; cf. [17]. First, each multipole has, in general, an infinite number of resonances, associated with $p=0,1,2, \ldots$. Actually, this feature is nothing but a direct consequence of the well-known oscillatory nature of the Riccati-Bessel functions. Second, owing to the additional degree of freedom related to variations of $p$, there is the multiple degeneracy of the resonances. Specifically, the resonances with different multipolarity $n$ occur at one and the same value of $m$, provided that for these resonances the variation of $n$ is compensated by the corresponding variation of $p$; i.e., $n_{1}+2 p_{1}=n_{2}+2 p_{2}$ for two electric resonant modes.

Next, at a given $n$ the points of the $n$th electric resonances correspond to those of the $(n+1)$ th magnetic and vice versa. Note also that at a fixed $n$ the points of resonances of one type (i.e., either electric, or magnetic) are situated in the $m$ axis just in the middle of the spacing between the points of the other type, so that the maxima for the electric modes correspond to the minima of magnetic and the other way around; see Eqs. (3.31) and (3.32).

In Fig. 1 we plot the dependence of the first two electric $d_{1,2}$ and magnetic $c_{1,2}$ internal coefficients vs size parameter $x$ and refractive index $m$, calculated according to the exact Mie solution. In this figure we also plot asymptotic resonance conditions (3.31) and (3.32). The agreement between the positions of the resonances according to the exact solution and approximate conditions (3.31) and (3.32), is surprisingly good.

It should be stressed, however, that the obtained results do not imply a strong overlap of the resonances yet. The point is that the right-hand side of Eq. (3.22) and the corresponding expression in Eq. (3.30) are just the first terms of the asymptotic expansions of the Riccati-Bessel functions in powers of small $1 /(m x)$. Higher-order terms dropped in these equations bring about the mismatches between the points of the resonances for different modes. To claim the strong overlap of the resonances, we must make sure that the mismatches are smaller than the corresponding linewidths. Let us proceed to a discussion of these issues. 

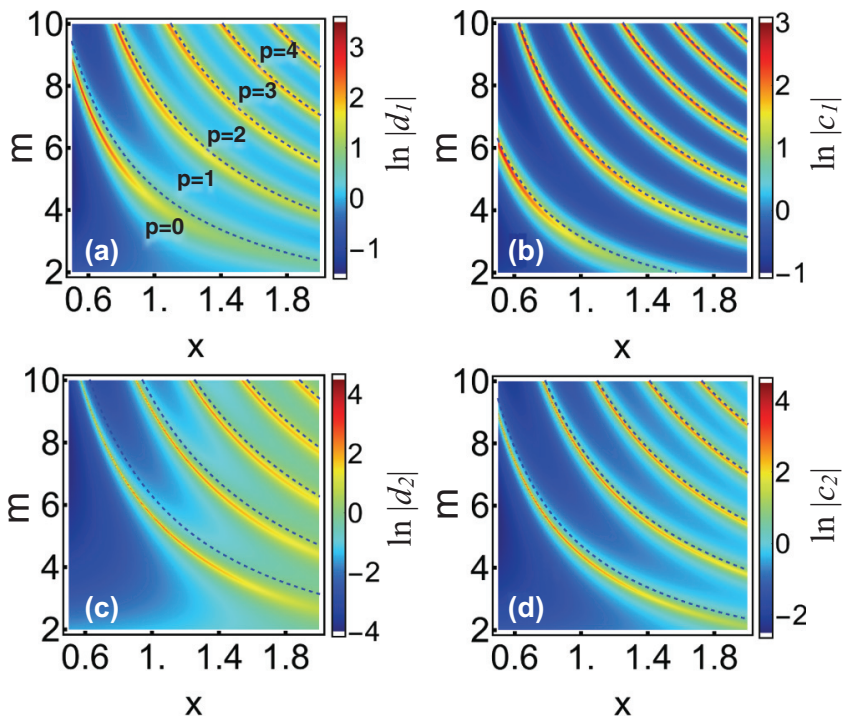

FIG. 1. Dependence of electric $d_{1,2}$ (a),(c) and magnetic $c_{1,2}$ (b),(d) internal coefficients vs size parameter $x$ and refractive index $m$ calculated according to the exact Mie solution. Dashed lines indicate the asymptotic resonance conditions in limit $m \rightarrow \infty$; see Eqs. (3.31) and (3.32) at $p=0,1,2,3$. Despite that the employed values of $m$ are not so large, the agreement between the dashed lines and the positions of the actual resonances is very good.

\section{LINEWIDTH AND LINE SHAPE}

To understand the meaning of the existence of the limit at $m \rightarrow \infty$ for scattering coefficients $a_{n}$ and $b_{n}$, and its absence for internal ones, $d_{n}$ and $c_{n}$, we have to inspect Eqs. (3.12)-(3.15) more carefully.

First of all, note that direct calculations of $a_{n}$ based upon Eqs. (3.10), (3.16), and (3.17) at the points of the resonances defined by Eq. (3.21) bring about the uncertainty of the type $0 / 0$. To resolve the line shape, let us consider a small departure of the refractive index from a certain resonant value: $\delta m=m-m^{(\mathrm{res})}$, where $m^{(\mathrm{res})}$ satisfies Eq. (3.21). Here and in what follows, for simplicity of notation, we use $m^{\text {(res) }}$ for $m_{n, p}^{(\mathrm{res}, E)}$. Then, bearing in mind that in the vicinity of the resonance $\psi_{n}\left[\left(m^{(\mathrm{res})}+\delta m\right) x\right] \cong \psi_{n}^{\prime}\left(m^{(\mathrm{res})} x\right) x \delta m$, we readily obtain the following formulas for $F_{n}^{(a)}, G_{n}^{(a)}$ :

$$
\begin{gathered}
F_{n}^{(a)} \cong \psi_{n}^{\prime}\left(m^{(\mathrm{res})} x\right)\left\{m^{(\mathrm{res})} \psi_{n}^{\prime}(x) x \delta m-\psi_{n}(x)\right\}, \\
G_{n}^{(a)} \cong-\psi_{n}^{\prime}\left(m^{(\mathrm{res})} x\right)\left\{m^{(\mathrm{res})} \chi_{n}^{\prime}(x) x \delta m-\chi_{n}(x)\right\} .
\end{gathered}
$$

It is seen from Eqs. (3.10), (4.1), and (4.2) that scattering coefficient reaches its maximum (the points of the constructive interference), $a_{n}=1$, at

$$
\delta m_{G}^{(a)} \cong \frac{1}{x m^{(\mathrm{res})}} \frac{\chi_{n}(x)}{\chi_{n}^{\prime}(x)}
$$

and minimal value, $a_{n}=0$ (the points of the destructive interference), at

$$
\delta m_{F}^{(a)} \cong \frac{1}{x m^{(\mathrm{res})}} \frac{\psi_{n}(x)}{\psi_{n}^{\prime}(x)}
$$

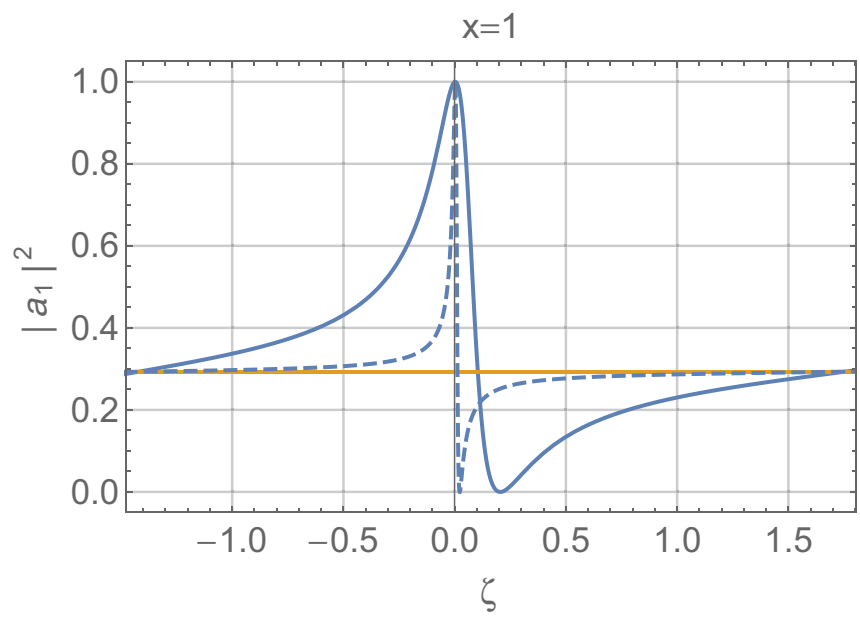

FIG. 2. Contraction of the resonance lines for $\left|a_{1}\right|^{2}$ with an increase in $m ; x=1 ; \zeta=m-m^{(\text {res })}$. Here $m^{\text {(res) }}=10.75$ (solid line) and $m^{\text {(res) }}=111.5$ (dashed line). A yellow horizontal line corresponds to $a_{1}^{(\mathrm{PEC})}$.

The corresponding expressions for $b_{n}$ read

$$
F_{n}^{(b)} \cong m^{(\mathrm{res})} \psi_{n}(x) \psi_{n}^{\prime \prime}\left(m^{(\mathrm{res})} x\right) x \delta m-\psi_{n}^{\prime}(x) \psi_{n}\left(m^{(\mathrm{res})} x\right),
$$

$$
G_{n}^{(b)} \cong-m^{(\mathrm{res})} \chi_{n}(x) \psi_{n}^{\prime \prime}\left(m^{(\mathrm{res})} x\right) x \delta m+\chi_{n}^{\prime}(x) \psi_{n}\left(m^{(\mathrm{res})} x\right),
$$

$$
\begin{aligned}
& \delta m_{G}^{(b)} \cong \frac{1}{x m^{(\mathrm{res})}} \frac{\chi_{n}^{\prime}(x) \psi_{n}\left(m^{(\mathrm{res})} x\right)}{\chi_{n}(x) \psi_{n}^{\prime \prime}\left(m^{(\mathrm{res})} x\right)}, \\
& \delta m_{F}^{(b)} \cong \frac{1}{x m^{(\mathrm{res})}} \frac{\psi_{n}^{\prime}(x) \psi_{n}\left(m^{(\mathrm{res})} x\right)}{\psi_{n}(x) \psi_{n}^{\prime \prime}\left(m^{(\mathrm{res})} x\right)}
\end{aligned}
$$

where now $m^{(\mathrm{res})}$ stands for $m_{n, p}^{(\mathrm{res}, H)}$, satisfying Eq. (3.29). Note that $\psi_{n}\left(m^{(\text {res })} x\right) / \psi_{n}^{\prime \prime}\left(m^{(\text {res })} x\right) \cong-1$ at $m^{(\text {res })} x>2 n$; see Eqs. (3.22) and (3.23).

The properties of the resonance lines discussed in the previous paragraphs are typical for the Fano profiles [27]. However, presently we are interested in the linewidth of these profiles solely. A detailed discussion of the line shape of the Fano resonances for the problem in question is produced in Sec. V.

The obtained results explain the mentioned convergence of $a_{n}$ to the $m$-independent form at $m \rightarrow \infty$ : At an increase in $m$ the width of the resonance line $\left|\delta m_{G}-\delta m_{F}\right|$ contracts as $1 / \mathrm{xm}^{\text {(res) }}$, while the amplitude of the resonances and spacing between two adjacent maxima (minima), both remain $m$ independent. Asymptotically, at $m \rightarrow \infty$ the maxima and minima merge with each other, and the resonance profile vanishes. An example of such a process is shown in Fig. 2.

Now let us inspect coefficients $d_{n}$. As it follows from Eqs. (3.2) and (3.19)

$$
a_{n}=a_{n}^{(\mathrm{PEC})}-\frac{\psi_{n}^{\prime}(m x)}{m \xi_{n}^{\prime}(x)} d_{n} .
$$


Then, it is convenient to introduce the normalized coefficient

$$
\tilde{d}_{n}=\frac{\psi_{n}^{\prime}(m x)}{m \xi_{n}^{\prime}(x)} d_{n} \equiv \frac{i}{m \frac{\psi_{n}(m x)}{\psi_{n}^{\prime}(m x)}-\frac{\xi_{n}(x)}{\xi_{n}^{\prime}(x)}} \frac{1}{\xi_{n}^{\prime 2}(x)} .
$$

Next, utilizing the identity

$$
\left|\xi_{n}^{\prime 2}(x) \operatorname{Im}\left[\frac{\xi_{n}(x)}{\xi_{n}^{\prime}(x)}\right]\right| \equiv 1
$$

(see Appendix), its modulus may be presented as

$$
\left|\tilde{d}_{n}\right|^{2}=\frac{1}{\left[m \frac{\psi_{n}(m x)}{\psi_{n}^{\prime}(m x)}-A_{n}^{(d)}(x)\right]^{2} B_{n}^{(d) 2}(x)+1},
$$

where

$$
A_{n}^{(d)}(x)=\operatorname{Re}\left[\frac{\xi_{n}(x)}{\xi_{n}(x)^{\prime}}\right] ; \quad B_{n}^{(d)}(x)=\left|\xi_{n}^{\prime 2}(x)\right| \equiv\left|\xi_{n}^{\prime}(x)\right|^{2} .
$$

The conclusion, which immediately follows from Eq. (4.13), is that the resonant values of $\left|\tilde{d}_{n}\right|^{2}$ equal unity and the resonances are achieved at the values of $m$, satisfying the equation

$$
m \frac{\psi_{n}(m x)}{\psi_{n}^{\prime}(m x)}=A_{n}^{(d)}(x) .
$$

We should emphasize that the resonance condition stipulated by Eq. (4.14) is an exact result, valid at any $m$ and $x$. To obtain the line shape in the proximity of $m=m^{\text {(res) }}$, with $m^{\text {(res) }}$ defined by Eq. (3.21), as usual, we have to expand the argument of $\psi_{n}(m x)$ in powers of a small $\delta m=m-m^{(\text {res })}$, so that $\psi_{n}(m x) \cong \psi_{n}^{\prime}\left(m^{(\mathrm{res})} x\right) x \delta m$. Then the general expression, Eq. (4.12), is reduced to the following simple form:

$$
\left|\tilde{d}_{n}\right|^{2}=\frac{1}{\left[m^{(\mathrm{res})} x \delta m-A_{n}^{(d)}(x)\right]^{2} B_{n}^{(d) 2}(x)+1} .
$$

It is a typical Lorentzian profile with $\operatorname{Max}\left\{\left|\tilde{d}_{n}\right|^{2}\right\}=1$ at

$$
\delta m_{\left|d_{n}\right|^{2}}^{(\mathrm{res})}=\frac{A_{n}^{(d)}(x)}{m^{(\mathrm{res})} x}
$$

and the half-maximum linewidth (FWHM)

$$
\gamma_{\left|d_{n}\right|^{2}}=\frac{2}{m^{(\mathrm{res})} x B_{n}^{(d)}(x)} .
$$

Note that Eq. (4.16) gives a mismatch of the order of $1 / m$ between the positions of the resonance points defined according to Eq. (3.21) and the ones corresponding to the actual maxima of $\left|d_{n}\right|^{2}$.

It seems we have encountered a paradox. On one side, we have obtained that, in contrast to the outer problem, the inner one does not have a definite limit at $m \rightarrow \infty$; see Eq. (3.18).

On the other side, $\tilde{d}_{n}=a_{n}^{(\mathrm{PEC})}-a_{n}$; see Eqs. (4.9) and (4.10). This equality means that since $a_{n} \underset{m \rightarrow \infty}{\longrightarrow} a_{n}^{(\mathrm{PEC})}$, coefficient $\tilde{d}_{n}$ should vanish in this limit. The latter reasoning agrees well with that following from the just obtained for $\tilde{d}_{n}$ line shape. Indeed, $\operatorname{Max}\left\{\left|\tilde{d}_{n}\right|^{2}\right\}$ always equals unity, while the linewidth for $\left|\tilde{d}_{n}\right|^{2}$ vanishes at $m \rightarrow \infty$ as $1 / m^{\text {(res) }}$ and the value of $\left|\tilde{d}_{n}\right|^{2}$ at off-resonance regions tends to zero as $1 / \mathrm{m}^{2}$; see Eq. (4.12).

Then, at $m \rightarrow \infty$ the resonance lines become infinitesimally narrow and the entire profile $\left|\tilde{d}_{n}(m)\right|^{2}$ vanishes. Finally, because the difference between $\tilde{d}_{n}$ and $d_{n}$ is just in the multiplicative scaling factor the same conclusion, seemingly, may be applied to the profile $\left|d_{n}(m)\right|^{2}$.

However, one should be careful here, because the scaling factor itself depends on $m$. Since $\max \left|\tilde{d}_{n}\right|^{2}=1$, the maximal value of $\left|d_{n}\right|^{2}$ increases as $m^{\text {(res) } 2}$; see Eq. (4.10). It means that the total area under a given resonance line increases with an increase in $m^{(\mathrm{res})}$ linearly in $m^{(\mathrm{res})}$, see Eq. (4.17), making (in contrast to $a_{n}$ ) the resonances more pronounced.

Nevertheless, it does not mean the absence of any universality in the profile $\left|d_{n}(m)\right|^{2}$ at $m \rightarrow \infty$. Let us consider $a$ part of the profile from its bottom to any fixed value $D_{n}^{2}$. According to Eq. (4.10),

$$
d_{n}=\frac{m \xi_{n}^{\prime}(x)}{\psi_{n}^{\prime}(m x)} \tilde{d}_{n} \equiv \frac{m\left[\psi_{n}^{\prime}(x)-i \chi_{n}^{\prime}(x)\right]}{\psi_{n}^{\prime}(m x)} \tilde{d}_{n} .
$$

Then, bearing in mind that in the Fraunhofer regime for the discussed resonances $\left|\psi_{n}^{\prime}\left(m^{(\text {res })} x\right)\right|=1$ [see Eqs. (3.22) and (3.31)], the width of the line at this distance from the bottom $\left(\gamma_{D}\right)$ is given by the difference $\left|\delta m_{1}-\delta m_{2}\right|$, where $\delta m_{1,2}$ are the two roots of the equation

$$
\frac{m^{(\mathrm{res}) 2}\left[\psi_{n}^{\prime 2}(x)+\chi_{n}^{\prime 2}(x)\right]}{\left[m^{(\mathrm{res})} x \delta m-A_{n}^{(d)}(x)\right]^{2} B_{n}^{(d) 2}(x)+1}=D^{2} .
$$

Trivial calculations show that at $D^{2} \ll m^{(\mathrm{res}) 2}\left[\psi_{n}^{\prime 2}(x)+\chi_{n}^{\prime 2}(x)\right]$ the quantity $\gamma_{D}$ converges to the following $m$-independent expression:

$$
\gamma_{D} \cong \frac{2 \sqrt{\psi_{n}^{\prime 2}(x)+\chi_{n}^{\prime 2}(x)}}{D B(x) x} .
$$

This asymptotic is valid for any $D$ satisfying the aforementioned constraint. It means that at large $m$ the shape of the bottom part of the resonance line becomes universal. An example of this universality for $n=1$ and $x=1$ is shown in Fig. 3.

Thus, instead of the vanishing (as it should be for a PEC) at $m \rightarrow \infty$, the resonance lines for coefficient $\left|d_{n}\right|^{2}$ at the bottom converge to a certain universal form, while the maximal value of $\left|d_{n}\right|^{2}$ at the peak of the resonance increases as $m^{\text {(res) } 2}$ and the half-maximum line width contracts as $1 / m^{(\text {res })}$. The behavior of $c_{n}$ is quite analogous to that of $d_{n}$. The expressions describing this behavior are presented below for reference:

$$
\begin{gathered}
b_{n}=b_{n}^{(\mathrm{PEC})}-\tilde{c}_{n}, \quad \tilde{c}_{n} \equiv \frac{\psi_{n}(m x)}{m \xi_{n}(x)} c_{n} \\
\left|\tilde{c_{n}}\right|^{2}=\frac{1}{\left[m \frac{\psi_{n}^{\prime}(m x)}{\psi_{n}(m x)}-A_{n}^{(c)}(x)\right]^{2} B_{n}^{(c) 2}(x)+1} \\
\approx \frac{1}{\left[\frac{\psi_{n}^{\prime \prime}\left(m^{(\mathrm{res})} x\right)}{\psi_{n}\left(m^{(\mathrm{res})} x\right)} m^{(\mathrm{res})} x \delta m-A_{n}^{(c)}(x)\right]^{2} B_{n}^{(c) 2}(x)+1}, \\
\delta m_{\left|c_{n}\right|^{2}}^{(\mathrm{res})} \frac{A_{n}^{(c)}(x) \psi_{n}\left(m^{(\mathrm{res})} x\right)}{m^{(\mathrm{res})} x \psi_{n}^{\prime \prime}\left(m^{(\mathrm{res})} x\right)} \\
\gamma_{\left|c_{n}\right|^{2}}=\frac{2}{m^{(\mathrm{res})} x B_{n}^{(c)}(x)}\left|\frac{\psi_{n}\left(m^{(\mathrm{res})} x\right)}{\psi_{n}^{\prime \prime}\left(m^{(\mathrm{res})} x\right)}\right| .
\end{gathered}
$$




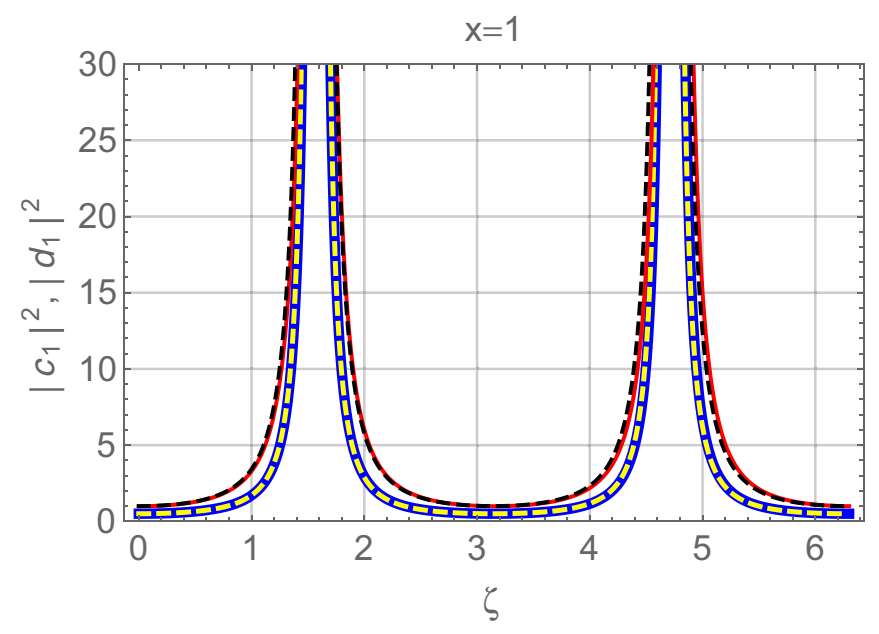

FIG. 3. Independence of the bottom parts of the resonance lines from $m^{\text {(res) }}$ for profiles $\left|c_{1}\right|^{2}$ and $\left|d_{1}\right|^{2}$ at $x=1$ and large values of $m^{\text {(res) }} ; \zeta=m-m^{\text {(res) }}$. Here profiles $\left|c_{1}(\zeta)\right|^{2}$ and $\left|d_{1}(\zeta)\right|^{2}$ are presented at $m^{(\mathrm{res})}=14.1019 \ldots$ (thick blue solid line), $m^{(\mathrm{res})}=$ $1013.1631 \ldots$ (thick yellow dashed line) and $m^{\text {(res) }}=15.5792 \ldots$ (red solid line), $m^{\text {(res) }}=1014.7325 \ldots$ (black dashed line), respectively. Three orders of magnitude increase in $m^{\text {(res) }}$, practically, does not affect the shape of the lines. Note the perfect coincidence of the corresponding lines, even in the off-resonance regions, despite that Eq. (4.20) is valid only in the vicinity of the points of the resonances and, generally speaking, cannot be applied to describe the line shape far from them.

Here $m^{(\text {res })}$ is defined according to Eq. (3.29),

$$
A_{n}^{(c)}(x)=\operatorname{Re}\left[\frac{\xi_{n}^{\prime}(x)}{\xi_{n}(x)}\right] ; \quad B_{n}^{(c)}(x)=\left|\xi_{n}^{2}(x)\right| \equiv\left|\xi_{n}(x)\right|^{2},
$$

and to obtain Eq. (4.22) the identity

$$
\left|\xi_{n}^{2}(x) \operatorname{Im}\left[\frac{\xi_{n}^{\prime}(x)}{\xi_{n}(x)}\right]\right| \equiv 1,
$$

whose proof is completely analogous to that for Eq. (4.11), has been employed.

Let us make numerical estimates. At $x=1.3$ and $m \approx 3-5$ (typical values for a number of semiconductors in the visible and IR diapasons) the resonant $\left|d_{1}\right|^{(\text {res })} \approx 3.01\left(m^{(\text {res })} \approx 3.33\right)$; $\left|d_{2}\right|^{(\mathrm{res})} \approx 11.78\left(m^{(\mathrm{res})} \approx 4.27\right) ;\left|d_{3}\right|^{(\mathrm{res})} \approx 93.59\left(m^{(\mathrm{res})} \approx 5.30\right)$. It gives an estimate for the range of the growth of the electric field inside the particle with respect to the incident wave. The corresponding values for the volume density of the electromagnetic energy will be the squire of these values.

To conclude this section, a certain important remark should be made. Though in a formal theoretical study it is convenient to inspect the resonances at varying $m$ and fixed values of the other problem parameters, such an approach seems completely irrelevant from the physical viewpoint. Indeed, while in an actual experiment it is rather easy to change the value of the size parameter, $x$ just varying the wavelength of the incident light, it is very difficult to change $m$. The refractive index is a fixed property of a given material. To change it, either materials with strong dispersion should be employed, or one has to have a set of particles with the same size made of different materials with their refractive indices changing in small steps. Both the options look unrealistic.

Thus, it seems our study is meaningless. Fortunately, this is not the case. The point is that the actual parameter of the theory is the product, $\rho=m x$. The problem in question corresponds to $\rho \gg 1$, while the spacing between two sequential resonances $\delta \rho=\pi$; see Eqs. (3.31) and (3.32). Now, if instead of variations of $m$ at a fixed $x$, we have variations of $x$ at a fixed $m$ to cover the distance between the two sequential resonances we have to consider departures of $x$ from a resonant value $x^{\text {(res) }}$ [defined by the same conditions, Eqs. (3.21) and (3.29)] of the order $\delta x \sim 1 / m \ll 1$. It means that in slowly varying functions of $x$ solely, namely in $\xi_{n}(x), \psi_{n}(x)$, and $\chi_{n}(x)$, we may neglect these small variations of $x$, replacing these functions by their values at $x=x^{(\text {res })}$. The only remaining step is to replace $\delta \rho=x \delta m$ by $\delta \rho=m \delta x$. Then, all the expressions obtained in this section and in what follows are readily recalculated for the case of varying $x$ and fixed $m$. More detailed discussion of this issue is presented in Sec. VIII.

\section{FANO RESONANCES IN PARTIAL-WAVE SCATTERING}

Up to now we have inspected the modula of the scattering coefficients. Let us focus on their phases. Following the approach, described in monograph [17], it makes sense to introduce a real angle $\Delta_{n}^{(a)}$ according to the expression

$$
\tan \Delta_{n}^{(a)} \equiv \frac{F_{n}^{(a)}}{G_{n}^{(a)}} .
$$

In this case $a_{n}$ can be written in the following form:

$$
a_{n}=\frac{\tan \Delta_{n}^{(a)}}{\tan \Delta_{n}^{(a)}+i}=\frac{i}{2}\left(1-e^{2 i \Delta_{n}^{(a)}}\right) .
$$

We recall that $a_{n}$ may be presented as a sum of two terms: $a_{n}^{(\mathrm{PEC})}$ and $\tilde{d}_{n}=d_{n} \psi_{n}^{\prime}(m x) /\left[m \xi_{n}^{\prime}(x)\right]$; see Eq. (4.9). Here the first term does not depend on $m$, while the second is a sharp function of $m$. Then, it is convenient to split $\Delta_{n}^{(a)}$ into two parts in the same manner, namely to present it as

$$
\Delta_{n}^{(a)} \equiv \Delta_{n}^{(a, \mathrm{PEC})}+\Delta_{n}^{(a, \text { res })},
$$

where $\Delta_{n}^{(a, \mathrm{PEC})}$ is defined for a PEC quite analogous to the definition of the just introduced $\Delta_{n}^{(a)}$ for a particle with arbitrary $m$, i.e.,

$$
\tan \Delta_{n}^{(a, \mathrm{PEC})} \equiv \frac{F_{n}^{(a, \mathrm{PEC})}}{G_{n}^{(a, \mathrm{PEC})}}=-\frac{\psi_{n}^{\prime}(x)}{\chi_{n}^{\prime}(x)} ;
$$

see Eq. (3.20). Next, the quantity $\Delta_{n}^{(a, \text { res })}$ is defined by the identity

$$
\begin{aligned}
\tan \Delta_{n}^{(a)} & \equiv \tan \left(\Delta_{n}^{(a, \text { res })}+\Delta_{n}^{(a, \text { PEC })}\right) \\
& =\frac{\tan \Delta_{n}^{(a, \text { res })}+\tan \Delta_{n}^{(a, \text { PEC })}}{1-\tan \Delta_{n}^{(a, \text { res })} \tan \Delta_{n}^{(a, \text { PEC })}} .
\end{aligned}
$$

Taking into account Eqs. (3.7), (3.10), and (5.4), after some algebra it is possible to show that to satisfy Eq. (5.5) identically, the tangent of $\Delta_{n}^{(a, \text { res })}$ must be

$$
\tan \Delta_{n}^{(a, \text { res })}=-\frac{\psi_{n}^{\prime}(m x)}{F_{n}^{(a, \text { PEC })} F_{n}^{(a)}+G_{n}^{(a, \mathrm{PEC})} G_{n}^{(a)}} .
$$


If now we introduce the notations

$$
\epsilon_{n}^{(a)} \equiv-\cot \Delta_{n}^{(a, \text { res })}, \quad q_{n}^{(a)} \equiv-\cot \Delta_{n}^{(a, \mathrm{PEC})}=\frac{\chi_{n}^{\prime}(x)}{\psi_{n}^{\prime}(x)},
$$

expression Eq. (5.2) for $a_{n}$ may be rewritten as follows:

$$
a_{n}=\frac{\epsilon_{n}^{(a)}+q_{n}^{(a)}}{\epsilon_{n}^{(a)}+q_{n}^{(a)}-i\left(\epsilon_{n}^{(a)} q_{n}^{(a)}-1\right)} .
$$

Then,

$$
\left|a_{n}\right|^{2}=\frac{\left(\epsilon_{n}^{(a)}+q_{n}^{(a)}\right)^{2}}{\left(1+q_{n}^{(a) 2}\right)\left(1+\epsilon_{n}^{(a) 2}\right)} .
$$

Equation (5.9) is the conventional Fano profile, normalized to its maximal value $[27,40]$.

A similar treatment of $b_{n}$ gives rise to the expression for $\tan \Delta_{n}^{(b, \text { res })}$

$$
\tan \Delta_{n}^{(b, \text { res })}=\frac{\psi_{n}(m x)}{F_{n}^{(b, \mathrm{PEC})} F_{n}^{(b)}+G_{n}^{(b, \mathrm{PEC})} G_{n}^{(b)}} .
$$

The rest is the same as that for $a_{n}$. Specifically, introducing

$$
\epsilon_{n}^{(b)} \equiv-\cot \Delta_{n}^{(b, \text { res })}, \quad q_{n}^{(b)} \equiv-\cot \Delta_{n}^{(b, \mathrm{PEC})}=\frac{\chi_{n}(x)}{\psi_{n}(x)} .
$$

we arrive for $b_{n}$ and $\left|b_{n}\right|^{2}$ at the same type of the profiles given by Eqs. (5.8) and (5.9) with replacement $a \rightarrow b$.

Once again we encounter a discrepancy, this time with our own results. It seems that by means of just identical transformations we have proven that the profiles of $\left|a_{n}\right|^{2}$ and $\left|b_{n}\right|^{2}$ are of the Fano type always. On the other hand, it has been shown in our previous publication [41] that, at least in the case of small particles $(m x \ll 1)$, the Fano resonances in partial scattered coefficients cannot happen.

Naturally, the discrepancy, as usual, is a misapprehension. The fact is that the condition $m x \ll 1$ does not allow $m$ to be large enough to reach the point of the first Fano resonance.

Therefore, to prove that the profile [Eq. (5.9)] does correspond to the Fano line shape, we have to show that (i) in the specified range of variations of $m, \epsilon_{n}^{(a)}(m)$ reaches the values corresponding to the constructive $\left(\epsilon_{n}^{(a)}=1 / q_{n}^{(a)}\right)$ and destructive $\left(\epsilon_{n}^{(a)}=-q_{n}^{(a)}\right)$ conditions (i.e., the problem in question may exhibit the Fano resonances, indeed), and (ii) for a given Fano profile in the vicinity of both the constructive and destructive interferences $\epsilon$ is one and the same linear function of $m$. Regarding the former, the manifestation of the constructive and destructive resonances in the problem has been already shown in the previous sections of this paper and in other publications [18,24,26]. As for the latter, employing Eqs. (4.1) and (4.2), after simple calculations, it is possible to show that

$$
\begin{aligned}
\epsilon_{n}^{(a)}= & m^{(\mathrm{res})}\left[\psi_{n}^{\prime 2}(x)+\chi_{n}^{\prime 2}(x)\right] x \delta m \\
& -\psi_{n}(x) \psi_{n}^{\prime}(x)-\chi_{n}(x) \chi_{n}^{\prime}(x) .
\end{aligned}
$$

The corresponding expression for $\epsilon_{n}^{(b)}$ reads

$$
\begin{aligned}
\epsilon_{n}^{(b)}= & -m^{(\mathrm{res})} \frac{\psi_{n}^{\prime \prime}\left(m^{(\mathrm{res})} x\right)}{\psi_{n}\left(m^{(\mathrm{res})} x\right)}\left[\psi_{n}^{2}(x)+\chi_{n}^{2}(x)\right] x \delta m \\
& +\psi_{n}(x) \psi_{n}^{\prime}(x)+\chi_{n}(x) \chi_{n}^{\prime}(x) .
\end{aligned}
$$

We remind the reader that for the magnetic modes $m^{(\mathrm{res})}$ is defined by condition $\psi_{n}^{\prime}\left(m^{(\mathrm{res})} x\right)$ [see Eq. (3.29)]; i.e., it corresponds to local extrema of $\psi_{n}^{\prime}$. Now recall that $\psi_{n}^{\prime}(z)$ is a function oscillating about zero. Then its local extrema are either positive maxima or negative minima. In both cases ratio $\psi_{n}^{\prime \prime}\left(m^{(\text {res })} x\right) / \psi_{n}\left(m^{(\text {res })} x\right)$ is a negative quantity. Thus, the overall sign of the $\delta m$ prefactor in Eq. (5.13) is plus.

In the Fraunhofer regime, i.e., at $x>2 n$, Eqs. (5.12) and (5.13) are simplified dramatically, namely,

$$
\epsilon_{n}^{(a)} \cong \epsilon_{n}^{(b)} \cong m^{(\mathrm{res})} x \delta m .
$$

Here the asymptotic

$$
\chi_{n}(x) \cong \cos \left(x-\frac{n \pi}{2}\right)
$$

for $\chi_{n}(x)$ at $x \geqslant 2 n$ [42] has been used.

Equations (5.12) and (5.13) imply that in the vicinity of the resonances $\epsilon_{n}$ is a linear function of $\delta m$, indeed. Regarding the validity of this approximation up to the points of the extrema of the profile, it follows from Eqs. (4.3), (4.4), (4.7), and (4.8). Note that Eqs. (5.12) and (5.13) yield the characteristic scale for the Fano profiles in $\delta m$ of the order of $1 / m$, which agrees with the previous consideration of the linewidths; see Sec. IV.

It is worth mentioning that the accuracy of approximations (5.12) and (5.13) is surprisingly good. As an example, a comparison of Eqs. (5.7), (5.9), and (5.12) with the exact Mie solution for $\left|a_{1}\right|^{2}$ at $x=1$ in the vicinity of $m=4.5$ is shown in Fig. 4.

It is important that the asymmetry parameter $q_{n}^{(a, b)}$ in this case is expressed by simple formulas, Eqs. (5.7) and (5.11), in terms of $\psi_{n}(x)$ and $\chi_{n}(x)$; i.e., it depends just on the multipolarity of the scattered partial wave $n$, radius of the scattering sphere, and wavelength of the incident light (we remind that $x \equiv 2 \pi R / \lambda$ ) and does not depend on the reflective index of the particle $m$.

Note also that the expressions for $q_{n}^{(a, b)}$ may be obtained in a less formal way, too. According to the preceding analysis in the off-resonance regions $a_{n} \cong a^{(\mathrm{PEC})}$ and $b_{n} \cong b^{(\mathrm{PEC})}$. In

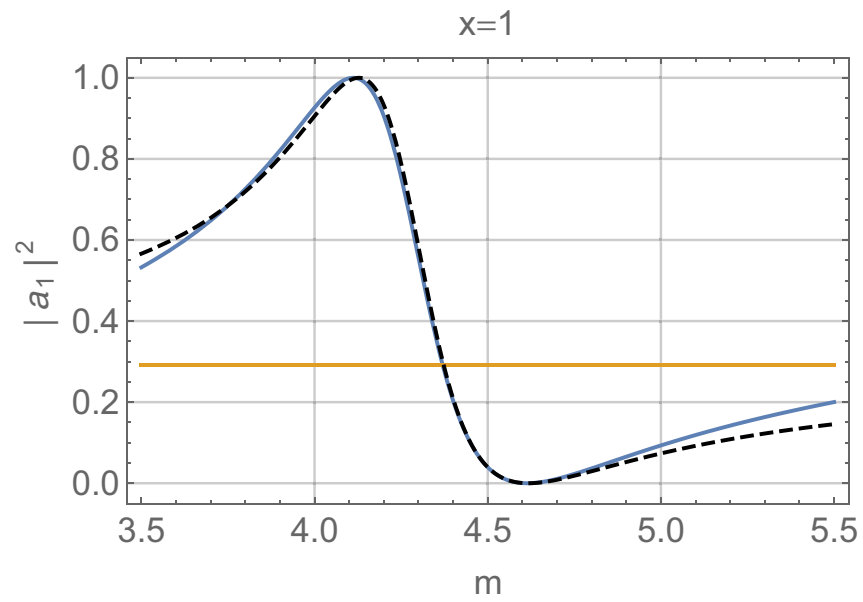

FIG. 4. Comparison of profile $\left|a_{1}(m)\right|^{2}$, given by the exact Mie solution (blue solid line), with that produced by Eqs. (5.7), (5.9); Eq. (5.12) (black dashed line), $x=1$. The yellow horizontal line corresponds to $a_{1}^{(\mathrm{PEC})}$. 


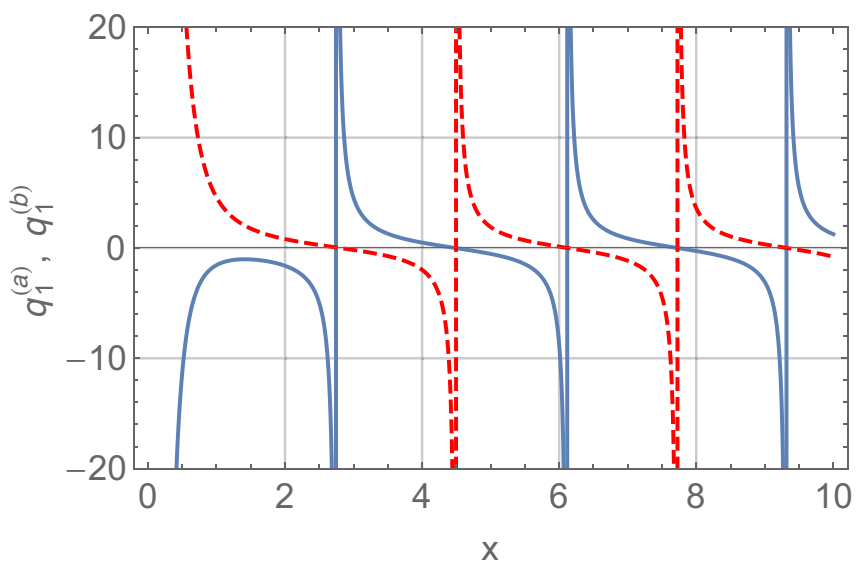

FIG. 5. Dependence of the asymmetry parameter $q$ on the size parameter $x: q_{1}^{(a)}(x)$ (solid blue line) and $q_{1}^{(b)}(x)$ (dashed red line), according to Eqs. (5.7) and (5.7).

terms of the Fano resonances, these regions correspond to the limit $\epsilon \rightarrow \infty$, when the Fano profile tends to $1 /\left(1+q^{2}\right)$; see Eq. (5.9). Equalizing $a^{(\mathrm{PEC})}$ to $1 /\left(1+q^{(a) 2}\right)$ and $b^{(\mathrm{PEC})}$ to $1 /\left(1+q^{(b) 2}\right)$, we again arrive at Eqs. (5.7) and (5.11) for $q_{n}^{(a, b)}$.

Let us discuss the dependence $q_{n}^{(a, b)}(x)$ in detail. At $x \geqslant 2 n$ we just can take the first term of the asymptotical expansions of the Riccati-Bessel functions. Then

$$
q_{n}^{(a)} \cong-\tan \left(x-\frac{n \pi}{2}\right), \quad q_{n}^{(b)} \cong \cot \left(x-\frac{n \pi}{2}\right) ;
$$

see Eqs. (3.22) and (5.15). Note that Eq. (5.16) yields $q_{n}^{(a)}=q_{n \pm 1}^{(b)}=q_{n+2}^{(a)}$.

In the opposite limit of small $x$, utilizing the known asymptotic expressions for the Bessel functions at a small value of the argument, we arrive at the following expressions for $q_{n}^{(a, b)}$ at $x \ll 1$,

$$
\begin{gathered}
q_{n}^{(a)} \cong-\frac{n}{n+1} \frac{2^{1+2 n} \Gamma\left(n+\frac{1}{2}\right) \Gamma\left(n+\frac{3}{2}\right)}{\pi x^{2 n+1}}, \\
q_{n}^{(b)} \cong \frac{2^{1+2 n} \Gamma\left(n+\frac{1}{2}\right) \Gamma\left(n+\frac{3}{2}\right)}{\pi x^{2 n+1}},
\end{gathered}
$$

where $\Gamma(z)$ stands for the Gamma function. As an example, the dependencies $q_{1}^{(a, b)}(x)$ in domain $0 \leqslant x \leqslant 10$ are shown in Fig. 5.

Both expressions, Eqs. (5.17) and (5.18), diverge as $1 / x^{2 n+1}$ at $x \rightarrow 0$. On the other hand, at large $q^{2}$ the Fano profile, Eq. (5.9), is reduced to the Lorentzian one:

$$
\frac{(\epsilon+q)^{2}}{\left(1+q^{2}\right)\left(1+\epsilon^{2}\right)} \underset{q \rightarrow \infty}{\longrightarrow} \frac{1}{1+\epsilon^{2}} .
$$

To understand the physical grounds for this reduction, we have to highlight the following. At the point of the constructive resonance $(\epsilon=1 / q)$, the Fano profile exhibits its maximal value, equal to unity. So does the Lorentzian profile. However, if for the latter its increase from the offresonance region also equals unity, for the former it equals the difference between unity and the background level; i.e.,
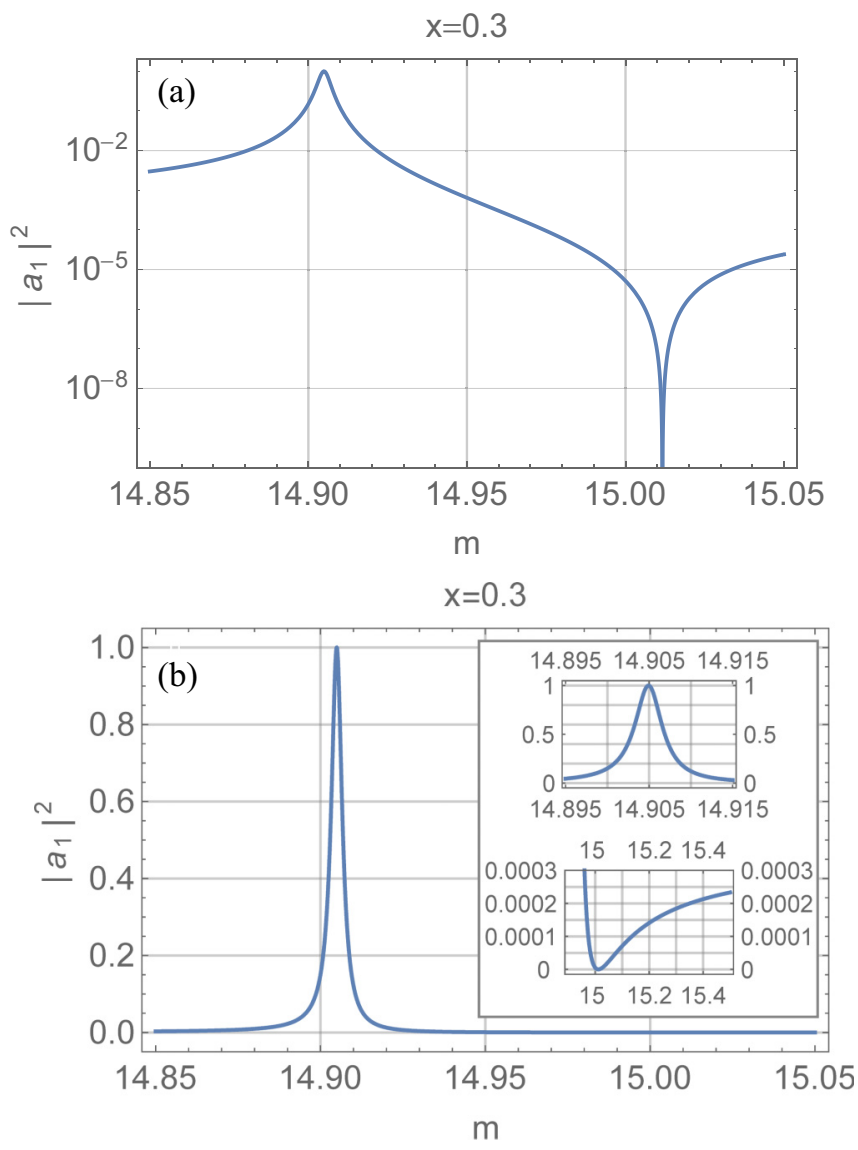

FIG. 6. Example of reduction of the Fano profile to Lorentzian at $x=0.3$. While the logarithmic plot of $\left|a_{1}(m)\right|^{2}$ (a) clearly exhibits the Fano line with the point of the constructive resonance $\left[\left|a_{1}(m)\right|^{2}=\right.$ $1]$ at $m \approx 14.905$ and the destructive resonance $\left[\left|a_{1}(m)\right|^{2}=0\right]$ at $m \approx 15.012$, a linear plot of the same dependence (b) is, practically, indistinguishable from the Lorentzian profile, centered about the point of the constructive resonance. The high-resolution line shape in the vicinity of the constructive and destructive resonances is shown in the inset. The background level $\left|a_{1}^{(\mathrm{PEC})}\right|^{2} \approx 0.0003404$. It corresponds to $q^{(a) 2} \approx 2937$.

$1-\left[1 /\left(1+q^{2}\right)\right]=q^{2} /\left(1+q^{2}\right)$. In the point of the destructive resonance the Fano profile drops below the background up to zero; that is to say, the amplitude of the corresponding modulation of the profile equals the background level $1 /(1+$ $\left.q^{2}\right)$. The ratio of the amplitudes of the two modulations of the profile (constructive to destructive) is just $q^{2}$. The larger $q^{2}$, the less pronounced the destructive resonance relative to constructive and the closer the Fano profile to Lorentzian.

If now we remember that the case $x \ll 1$ corresponds to a small particle and a small particle in the off-resonance regions is a weak scatterer, we immediately understand why the background level at $x \ll 1$ is small and the Fano profile is close to Lorentzian.

Thus, we have arrived at an important conclusion: Though, formally, the Mie resonances for the partial scattered waves of the outer problem for small particles still belong to the Fano type, actually, the corresponding line shape is very close to the Lorentzian; cf. [18]. An example of such a profile is presented in Fig. 6. 

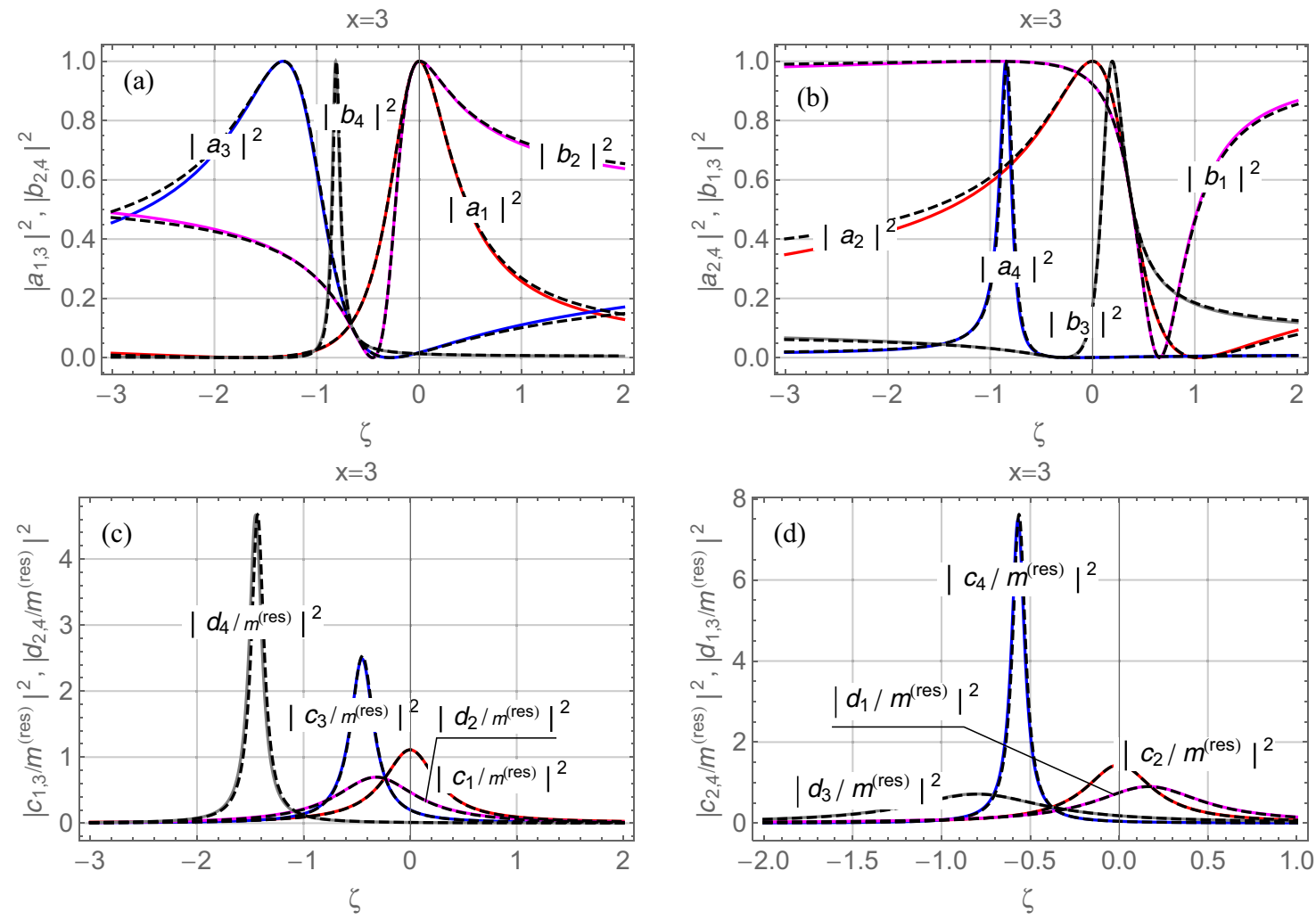

FIG. 7. Collapse of lines of the resonances to a single universal set, as a result of the scale transformation (6.1) and (6.2) at $x=3$; $\zeta=\left(m-m^{(\text {res })}\right) m^{\text {(res) }}$ : Color solid and black dotted lines respectively correspond to: (a) $m^{\text {(res) }}=9.944 \ldots$ and $m^{(\text {res })}=50.788 \ldots$ (local maxima of $\left|a_{1}\right|^{2}$ ); (b) $m^{\text {(res) }}=9.350 \ldots$ and $m^{\text {(res) }}=50.252 \ldots$ (local maxima of $\left|a_{2}\right|^{2}$ ); (c) $m^{\text {(res) }}=9.441 \ldots$ and $m^{(\text {res })}=50.263 \ldots$ (local maxima of $\left.\left|c_{1}\right|^{2}\right)$; (d) $m^{(\text {res })}=9.919 \ldots$ and $m^{(\text {res })}=50.783 \ldots$ (local maxima of $\left.\left|c_{2}\right|^{2}\right)$.

\section{SCALE INVARIANCE}

The results discussed in the previous sections give rise to a simple but significant conclusion. Namely, we have obtained that at any fixed $x$ and $n$ the profiles of $\left|a_{n}(m)\right|^{2}$ and $\left|b_{n}(m)\right|^{2}$ exhibit infinite sequences of the Fano resonances. All these resonances have one and the same $m$-independent value of $q$ [see Eq. (5.7)] and the characteristic scale, decreasing as $1 / m^{(\text {res })}$; see Eqs. (4.3) and (4.4). Since the shape of the Fano profile is completely defined by the value of $q$, the latter means that at a given $x$ all the Fano resonances are identical and may be reduced to a single universal form by the scale transformation

$$
\delta m \rightarrow m^{(\mathrm{res})} \delta m
$$

Regarding $\left|c_{n}\right|^{2}$ and $\left|d_{n}\right|^{2}$, at a fixed $m$ these profiles are Lorentzian and, therefore, also universal. The corresponding scale transformations are Eq. (6.1), supplemented with the rescaling of the coefficients

$$
c_{n} \rightarrow c_{n} / m^{(\mathrm{res})}, \quad d_{n} \rightarrow d_{n} / m^{(\mathrm{res})} .
$$

Finally, bearing in mind that the mismatches in the positions of the points of the resonances for modes with different $n$ are also scaled as $1 / m^{\text {(res) }}$, we obtain that the stipulated scale transformations should reduce the entire variety of the resonances (including the mutual position of the resonances with different $n$ ) to a single universal set. An example of such a collapse is shown in Fig. 7.

It is also seen from Fig. 7 that an increase in $n$ results in shifts of the resonances $\left|c_{n}\right|^{2}$ and $\left|d_{n}\right|^{2}$. The same is true for $\left|a_{n}\right|^{2}$ and $\left|b_{n}\right|^{2}$. However, for the Fano resonances [Figs. 7(a) and 7(b)] the increase in $n$ is accompanied with the change of the asymmetry parameter $q$ [see Eqs. (5.7) and (5.11)] and, hence, by the change of the corresponding line shape. Thus, to identify the shift one should look at the position of the local extrema. Note also that for the lines $\left|c_{n}\right|^{2}$ and $\left|d_{n}\right|^{2}$ the increase in $n$ results in an increase in the $Q$ factor of the resonances; see Figs. 7(c) and 7(d). These peculiarities are generic for the problem in question and valid for any $n$.

\section{DISSIPATIVE EFFECTS}

Recall now that the nondissipative limit discussed above is an idealized abstraction. In fact, dissipative losses always remain finite, as long as a real material is a concern. Then the natural question "How the obtained results are affected by the dissipative losses?" arises. In this section we answer the question. To this end, we have to introduce a complex refractive index,

$$
\hat{m}=m+i \kappa .
$$

Note that, actually, there are two cases: strong dissipation ( $m$ and $\kappa$ are of the same order of magnitude) and weak 
dissipation $(\kappa \ll m)$. The former case is trivial; the dissipation just suppresses the resonances. Thus, the most interesting is the weak-dissipation case, especially its limit of the extremely weak dissipation $(\kappa \ll 1)$, when the dissipative damping may compete with the small radiative one. It may give rise to the absorption resonances [43] and other effects similar to those at the anomalous scattering [44].

Examples of the extremely weak dissipation may be readily found among widely used semiconductors. For instance, at the wavelength of $532 \mathrm{~nm}$ (the second harmonic of a Nd:YAG laser) the complex refractive indices for gallium phosphide, silicon, and gallium arsenide are $\hat{m}_{\mathrm{GaP}}=3.4932+i 0.0026311$, $\hat{m}_{\mathrm{Si}}=4.1360+i 0.010205$, and $\hat{m}_{\mathrm{GaAs}}=4.1331+i 0.33609$, respectively [45]. In what follows all numerical examples, illustrating the developed theory, will be given for a particle made of gallium phosphate irradiated in a vacuum by a plane linearly polarized electromagnetic wave with $\lambda=532 \mathrm{~nm}$. The dispersion of $\hat{m}_{\mathrm{GaP}}$ will be neglected, since in the proximity of the specified wavelength it is rather weak. Let us discuss the weak-dissipation case in detail.

\section{A. Field within the particle}

It is convenient to begin the discussion with the effects of dissipation for the resonant excitation of the modes within the particle. As usual, first, we inspect the electric modes. Let us consider small complex departures of $\delta \hat{m}=\delta m+i \kappa$ from a purely real $m^{(\text {res })}$, defined by condition $\psi\left(m^{(\text {res })} x\right)=$ 0 . Expansion of $\psi(\hat{m} x)$ in powers of small $x \delta \hat{m}$ about the point $m^{(\text {res })} x$ results in the following trivial generalization of expression, Eq. (4.12), for $\left|\tilde{d}_{n}\right|^{2}$,

$\left|\tilde{d}_{n}\right|^{2}=\frac{1}{\left[m^{(\mathrm{res})} x \delta m-A_{n}^{(d)}(x)\right]^{2} B_{n}^{(d) 2}(x)+\left[1+\kappa B_{n}^{(d)}(x) m^{(\mathrm{res})} x\right]^{2}}$,

with the connection between $\left|\tilde{d}_{n}\right|^{2}$ and $\left|d_{n}\right|^{2}$,

$$
\left|d_{n}\right|^{2}=\frac{m^{2} B_{n}^{(d)}(x)}{\left|\psi_{n}^{\prime}(m x)\right|^{2}}\left|\tilde{d}_{n}\right|^{2}
$$

following from Eqs. (4.10) and (4.13). Equation (7.2) yields the linewidth

$$
\gamma_{\left|d_{n}\right|^{2}}^{(\kappa)}=2 \frac{1+\kappa B_{n}^{(d)}(x) m^{(\mathrm{res})} x}{B_{n}^{(d)}(x) m^{(\mathrm{res})} x} .
$$

The profile $\left|d_{n}\right|^{2}$ is maximized by the same $\delta m_{\left|d_{n}\right|^{2}}^{\text {(res) }}$, given by Eq. (4.16), but the maximal value now is different:

$$
\operatorname{Max}\left\{\left|d_{n}\right|^{2}\right\} \cong \frac{m^{(\mathrm{res}) 2} B_{n}^{(d)}(x)}{\left|\psi_{n}^{\prime}\left(m^{(\mathrm{res})} x\right)\right|^{2}\left[1+\kappa B_{n}^{(d)}(x) m^{(\mathrm{res})} x\right]^{2}} .
$$

It is clearly seen from Eq. (7.4) that there is a point of crossover $\left(m_{\mathrm{cr}}\right)$ from the nondissipative regime (at $m \ll m_{\mathrm{cr}}$ ) to dissipative (at $m \gg m_{\mathrm{cr}}$ ), where $m_{\mathrm{cr}}$ is a solution of the equation:

$$
\kappa m x B_{n}^{(d)}(x)=1 .
$$

If at $m \ll m_{\mathrm{cr}}$ the linewidth is determined by Eq. (4.17), at $m \gg m_{\mathrm{cr}}$ it converges to the universal $m$ - $x$ - $n$-independent value $2 \kappa$.
However, the most dramatic changes happen to the amplitude of the resonances. While in the nondissipative limit the amplitude of the profile $\left|d_{n}\right|^{2}$ at the resonance points at large $m$ increases as $m^{2}$ [see Eq. (4.18)], now the entire profile converges to the universal form

$$
\left|d_{n}\right|^{2} \underset{m \gg m_{\mathrm{cr}}}{\longrightarrow} \frac{1}{\left|\psi_{n}^{\prime}\left(m^{(\mathrm{res})} x\right)\right|^{2} x^{2} B_{n}^{(d)}(x)} \frac{1}{\delta m^{2}+\kappa^{2}},
$$

which becomes completely $m^{\text {(res) }}$-independent in the Fraunhofer regime, $m^{\text {(res) }} x \geqslant 2 n$ [we remind the reader that in this regime $\left|\psi_{n}^{\prime}\left(m^{(\text {res })} x\right)\right| \cong 1$.

Equation (7.7), together with the condition $\gamma_{\left|d_{n}\right|^{2}}^{(\kappa)} \cong 2 \kappa$ at $m \gg m_{\text {cr }}$ and Eq. (3.31), defining the positions of the resonant points on the $m$ axis, give rise to the conclusion that at $m \gg m_{\text {cr }}$ the entire profile $\left|d_{n}(m)\right|^{2}$ converges to a universal periodic function of $m$.

Coefficients $c_{n}$ may be inspected exactly in the same manner. The inspection yields the expressions similar to those obtained above for $d_{n}$. For example,

$$
\operatorname{Max}\left\{\left|c_{n}\right|^{2}\right\} \cong \frac{m^{(\mathrm{res}) 2} B_{n}^{(c)}(x)}{\left|\psi_{n}\left(m^{(\mathrm{res})} x\right)\right|^{2}\left[1-\kappa \frac{\psi^{\prime \prime}\left(m^{(\mathrm{res})} x\right)}{\psi\left(m^{(\mathrm{res})} x\right)} B_{n}^{(c)}(x) m^{(\mathrm{res})} x\right]^{2}} .
$$

The reader should not be confused with minus sign in front of the term with the dissipative constant $\kappa$, since, due to the factor $\psi^{\prime \prime}\left(m^{(\mathrm{res})} x\right) / \psi\left(m^{(\mathrm{res})} x\right)$, the actual sign in front of the dissipative term is plus; see the corresponding discussion after Eq. (5.13). The other expressions for $c_{n}$, analogous to those discussed above for $d_{n}$, are not presented here owing to the triviality of the corresponding calculations. They result in the behavior of $c_{n}$ quite similar to that for $d_{n}$.

\section{B. Dissipation and Fano resonances}

These peculiarities of $c_{n}$ and $d_{n}$ bring about a completely different scenario for the vanishing of the Fano resonances for coefficients $a_{n}$ and $b_{n}$ relative to that in the nondissipative case. For definiteness, let us focus on $a_{n}$. This coefficient is expressed in terms of $m$-independent $a_{n}^{(\mathrm{PEC})}$ and $m$-dependent $d_{n}$ according to Eq. (4.9). Thus, the entire $m$ dependence of $a_{n}$ is given by the second term in the right-hand side of Eq. (4.9). We remind the reader that in the nondissipative limit $\left|a_{n}\right|^{2}$ always vanishes at the points of the destructive Fano resonances and reaches unity at the points of the constructive resonances; see Fig. 2 and Eq. (5.9). Asymptotically, the points of the constructive and destructive resonances merge, while the modulations of $\left|a_{n}\right|^{2}$ remain the same: Each resonance forces $\left|a_{n}\right|^{2}$ to vary from zero to unity. Thus, the vanishing of the Fano resonances with an increase in $m$ occurs due to the contraction of the resonance lines.

In contrast, now at a finite $\kappa$ and large-enough $m$ the width of the resonances for $\left|a_{n}\right|^{2}$ becomes $m$ independent and equal to $2 \kappa$ [according to identity Eq. (4.9) the characteristic widths of the resonances for $a_{n}$ and $d_{n}$ have the same order of magnitude], while the amplitude of the modulations of $\left|a_{n}-a_{n}^{(\mathrm{PEC})}\right|^{2}$ decreases as $1 / \mathrm{m}^{2}$; see Eqs. (4.9) and (7.7). Thus, the vanishing of the Fano resonances (convergence of $a_{n}$ to $a_{n}^{(\mathrm{PEC})}$ ) occurs owing to the vanishing of the amplitude of the 
modulations of $a_{n}$. Crossover from the nondissipative scenario to dissipative again is determined by $m_{\mathrm{cr}}$; see Eq. (7.6). The behavior of $b_{n}$ is analogous.

Now note that the presented in Sec. V Fano profiles for $\left|a_{n}\right|^{2}$ and $\left|b_{n}\right|^{2}$ have been obtained from the exact Mie solution by the identical transformations. The transformations remain valid, no matter wether the permittivity is a purely real (the nondissipative limit) or complex (the case of finite dissipation) quantity. It provides us with a unique opportunity to generalize the Fano theory to dissipative cases, i.e., to extend rigorously the theory beyond its initial quantum-mechanical formulation, where just effective dissipation attributed to, e.g., various dephasing mechanisms, may be introduced only phenomenologically. Such a generalizations permits us to supplement the semiquantitative discussion of the dissipative effects, presented above, with a detailed study of the degradation of the Fano profile due to increase of dissipation. In addition, instead of the discussion of the evolution of the profile of $\left|a_{n}-a_{n}^{(\mathrm{PEC})}\right|^{2}$, which, according to Eqs. (4.9), (4.10), and (7.2), is purely Lorentzian, it makes possible to inspect the transformation of the actual Fano profiles for $\left|a_{n}\right|^{2}$ and $\left|b_{n}\right|^{2}$.

In agreement with what has been discussed above, the generalization is quite trivial: It corresponds to the replacement $\delta m \rightarrow \delta m+i \kappa$. This transformation does not affect the asymmetry parameter $q$, since it does not depend on $m$; see Eqs. (5.7) and (5.11). However, it does affect $\epsilon$, making it complex; see Eqs. (5.12) and (5.13).

Thus, in a dissipative case $\operatorname{Im} q \equiv 0, \epsilon=\epsilon^{\prime}+i \epsilon^{\prime \prime}$. Substitution of the complex $\epsilon$ into Eq. (5.8) brings about the desired profile, which after some algebra may be reduced to the form

$$
\left|a_{n}\right|^{2}=\frac{\left(\epsilon_{n}^{(a) \prime}+q_{n}^{(a)}\right)^{2}+\epsilon_{n}^{(a) \prime \prime 2}}{\left(1+q_{n}^{(a) 2}\right)\left[\epsilon_{n}^{(a) / 2}+\left(1+\epsilon_{n}^{(a) \prime \prime}\right)^{2}\right]} .
$$

Replacement $a \rightarrow b$ in Eq. (7.9) yields the corresponding generalized Fano profile for $\left|b_{n}\right|^{2}$.

Let us discuss briefly the properties of the obtained profiles. It is seen straightforwardly that Eq. (7.9) transforms into the conventional Fano profile [Eq. (5.9)] at $\epsilon^{\prime \prime} \rightarrow 0$ and that at $\left|\epsilon^{\prime}\right| \gg \epsilon^{\prime \prime}$ and $\left|\epsilon^{\prime}\right| \gg|q|$ it tends to the same limit $1 /\left(1+q^{2}\right)$. It is also possible to show that at any fixed $q$ and $\epsilon^{\prime}$ the maximum and minimum of profiles [Eq. (7.9)] are monotonically decreasing and increasing functions of $\epsilon^{\prime \prime}$, respectively.

Regarding the amplitude of the resonance, defined as the difference between the maximal and minimal values of the profile, as a function of $\epsilon^{\prime}$ at fixed values of the two other parameters, it is given by the expression

$$
\Delta_{\left|a_{n}\right|^{2}}=\frac{1}{\left(1+\epsilon^{\prime \prime}\right)^{2}} \sqrt{\frac{q^{2}+\left(1+2 \epsilon^{\prime \prime}\right)^{2}}{1+q^{2}}} .
$$

At $\epsilon^{\prime \prime} \ll 1$ the amplitude is close to unity, as it should be for the conventional, nondissipative Fano resonances. At $\epsilon^{\prime \prime} \gg 1$ there are two cases. If $|q|$ is of the order of unity, or smaller than that, the amplitude vanishes as $2 /\left(\epsilon^{\prime \prime} \sqrt{1+q^{2}}\right)$. If $|q| \gg 1$, there is an intermediate asymptotic: At $1 \ll \epsilon^{\prime \prime} \ll|q|$ the amplitude dies out as $1 / \epsilon^{\prime \prime 2}$ and only at $\epsilon^{\prime \prime} \gg|q|$ is this asymptotic replaced with $2 /\left(\epsilon^{\prime \prime}|q|\right)$.

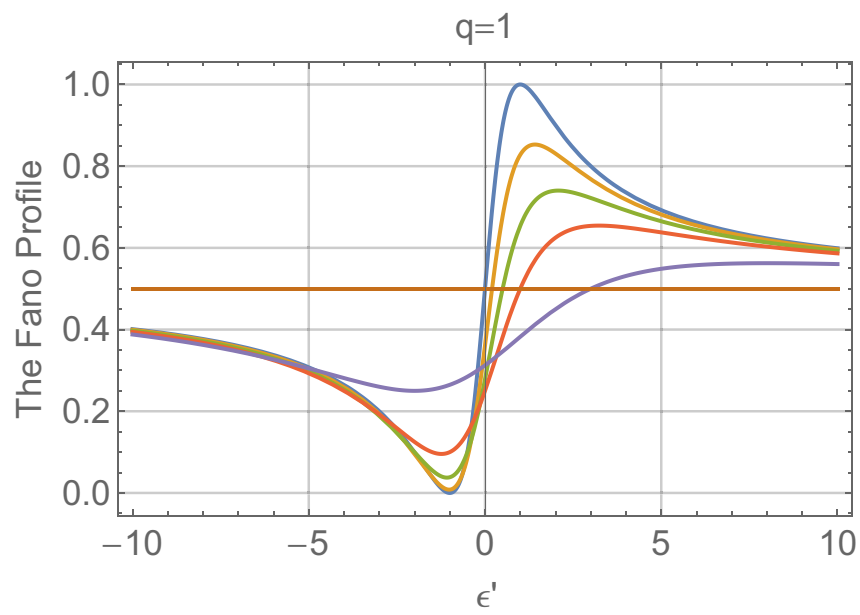

FIG. 8. Degradation of the Fano profile (7.9) due to increase of dissipation. The asymmetry parameter is fixed to $q=1$. The sharpest blue line corresponds to $\epsilon^{\prime \prime}=0$. For other profiles $\epsilon^{\prime \prime}$ equals $0.2,0.5$, 1.0, and 3.0, respectively. The horizontal brown line designates the background, which at $q=1$ equals $1 / 2$.

As for the linewidth, defined as the difference between the positions of the points of the maximum and minimum of the profile on the $\epsilon^{\prime}$ axis at fixed $\epsilon^{\prime \prime}$ and $q$, it is described by the formula

$$
\gamma_{\left|a_{n}\right|^{2}}=\frac{\sqrt{\left(1+q^{2}\right)\left[q^{2}+\left(1+2 \epsilon^{\prime \prime}\right)^{2}\right]}}{|q|} .
$$

At $\epsilon^{\prime \prime} \ll|q|$ the linewidth converges to the conventional Fano expression $\left(1+q^{2}\right) /|q|$. At $\epsilon^{\prime \prime} \gg|q|$ it equals $\left(1+2 \epsilon^{\prime \prime}\right) \sqrt{1+q^{2}} /|q|$.

The calculations yielding Eqs. (7.10) and (7.11) are trivial but cumbersome and are not presented here. An example of degradation of the Fano profile with an increase in $\epsilon^{\prime \prime}$ is shown in Fig. 8.

To relate these general analyses of the dissipation-affected Fano resonances to our problem, we must express $\epsilon^{\prime \prime}$ in terms of the known problem parameters. According to what has been discussed above and to Eqs. (5.12), (5.13), the desired expressions are as follows:

$$
\begin{gathered}
\epsilon_{n}^{(a) \prime \prime}=m^{(\mathrm{res})}\left[\psi_{n}^{\prime 2}(x)+\chi_{n}^{\prime 2}(x)\right] x \kappa, \\
\epsilon_{n}^{(b) \prime \prime}=-m^{(\mathrm{res})} \frac{\psi_{n}^{\prime \prime}\left(m^{(\mathrm{res})} x\right)}{\psi_{n}\left(m^{(\mathrm{res})} x\right)}\left[\psi_{n}^{2}(x)+\chi_{n}^{2}(x)\right] x \kappa .
\end{gathered}
$$

At $x \geqslant 2 n$

$$
\epsilon_{n}^{(a) \prime \prime} \cong \epsilon_{n}^{(b) \prime \prime} \cong m^{(\mathrm{res})} \kappa x ;
$$

cf. Eq. (5.14). It means that the role of the dissipative effects unlimitedly increases with an increase in $x$, no matter how small $\kappa$ is.

\section{RESONANCES AT FIXED REFRACTIVE INDEX AND VARYING SIZE PARAMETER}

In the preceding sections the resonances basically have been studied at a fixed $x$ (and $\kappa$ ) and varying $m$. On the other hand, as mentioned above, the most interesting from the experimental viewpoint is the dependence of the resonances on the size of 
the particle at a fixed value of the refractive index. In this case at any fixed $n$ an increase in $x$ again results in a cascade of resonances, whose positions are determined by the same conditions [Eqs. (3.21) and (3.29)], regarded now as equations for $x$.

At $m x \gg 1$, as long as the shape of a single resonance line is a concern, these two problem formulations (fixed $x$ at varying $m$ and fixed $m$ at varying $x$ ) are easily reduced to each other by the set of transformations $m^{\text {(res) }} \leftrightarrow m, x \leftrightarrow x^{(\text {res })}$, and $x \delta m \leftrightarrow m \delta x$; see the corresponding remark at the end of Sec. IV. However, this is not the case anymore if we are interested in rather a large range of variations of the size parameter.

For example, if we consider the Fano resonances for $\left|a_{n}\right|^{2}$ and $\left|b_{n}\right|^{2}$, the effective dissipative constant $\epsilon^{\prime \prime}$ increases linearly in $x$; see Eqs. (7.12) and (7.12). Eventually, it must result in suppression of the resonances at large-enough $x$. However, as shown in Sec. VII B, the dissipative effects depend substantially on the ratio of $\epsilon^{\prime \prime}$ to $|q|$. This ratio may have a rather complicated $x$-dependence, see Fig. 5 and Eqs. (5.7), (5.11), (7.12)-(7.14). Therefore, to answer the question about the transformation of the resonance profiles at large variations of $x$ is not a straightforward matter.

These arguments are illustrated by Fig. 9, where $\left|a_{1}(x)\right|^{2}$ and $\left|a_{1}^{(\mathrm{PEC})}(x)\right|^{2}$ for GaP at $\lambda=532 \mathrm{~nm}$ are plotted as functions of $x$. In Fig. 9(a), at moderate values of $x$ a cascade of the Fano resonances, whose asymmetry parameter varies with $x$ according to Eq. (5.7), is seen clearly. The cascade is quite analogous to those discussed in Refs. [24,26] for a dielectric cylinder. It is interesting to note that at the case presented in Fig. 9 at $x=4.483 \ldots,\left|a_{1}(x)\right|^{2}=\left|a_{1}^{(\mathrm{PEC})}(x)\right|^{2}=1$; i.e., for the given partial wave the scattering at this point is exactly the same as that for a perfectly reflecting sphere at the point of the dipole resonance.

In Fig. 9(b) the growth of $x$ already results in suppression of the resonances. In Fig. 9(c) the asymptotic profile of $\left|a_{1}\right|^{2}$ at large values of $x$ is presented. A further increase in $x$ does not affect this profile. The Fano resonances in Fig. 9(c) are fully suppressed, indeed. However, in contrast to what may be expected from the preceding analysis, the profile, which $\left|a_{1}\right|^{2}$ has converged to, is not that for $\left|a_{1}^{(\mathrm{PEC})}\right|^{2}$.

As we see below, the behavior of $\left|d_{n}\right|^{2}$ and $\left|c_{n}\right|^{2}$ at a large variation of $x$ is also rather unexpected. Thus, the transformations of the scattering coefficients at a fixed $\hat{m}$ and large variations of $x$ have their own peculiarities, which are worth studying. The study is produced in the present section.

\section{A. Scattered field: Fano resonances}

In the preceding paragraphs we have faced a puzzle: At a fixed $\hat{m}$ and $x \gg 1$ the profile $\left|a_{1}(x)\right|^{2}$ for GaP converges to a certain $\hat{m}$-independent profile, which, however, differs from $\left|a_{1}^{(\mathrm{PEC})}(x)\right|^{2}$. Direct calculations based upon the exact Mie solution for a particle with arbitrary large $m$ and small $\kappa$ indicate that this is quite a general feature for $\left|a_{n}\right|^{2}$ and $\left|b_{n}\right|^{2}$ at any $n$.

The puzzle is solved easily. To this end it is better to start from the very initial expressions for $a_{n}$ and $b_{n}$, namely Eqs. (3.10)-(3.15) and use the Fraunhofer approximation Eqs. (3.22) and (5.15).
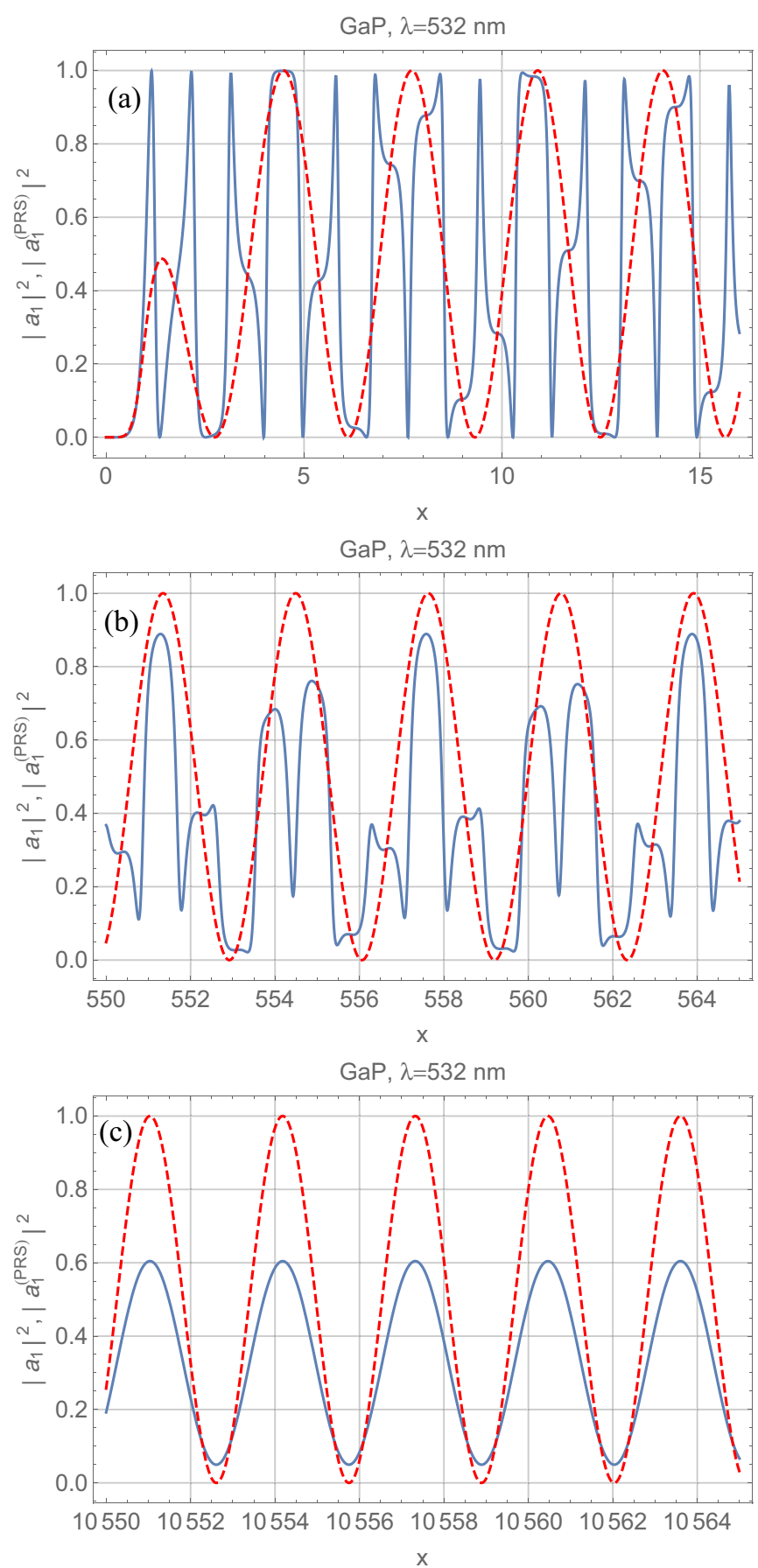

FIG. 9. Dependencies of $\left|a_{1}(x)\right|^{2}$ (blue solid line) and $\left|a_{1}^{(\mathrm{PEC})}(x)\right|^{2}$ (red dashed line) for $\mathrm{GaP}$ on the size parameter $x$ at various characteristic values of the latter. Profile (c) is asymptotic; it does not change its shape at further increase in $x$.

$$
\begin{aligned}
& \text { Then, at } \kappa x \\
& \qquad \begin{aligned}
\psi_{n}(\hat{m} x) & \cong \sin \left(m x+i \kappa x-\frac{n \pi}{2}\right) \\
& =\frac{e^{i \phi_{n}^{(m)}-\kappa x}-e^{-i \phi_{n}^{(m)}+\kappa x}}{2 i} \cong i \frac{e^{-i \phi_{n}^{(m)}+\kappa x}}{2},
\end{aligned}
\end{aligned}
$$

where $\phi_{n}^{(m)}$ stands for $m x-\frac{n \pi}{2}$. The rest is just trivial transformations of Eqs. (3.10)-(3.15). Exponents $\exp \left(-i \phi_{n}^{(m)}+\kappa x\right)$ 
in the numerators and denominators of the expressions for $a_{n}$ and $b_{n}$ are canceled. Since we are interested in the limit of large $x$, the Fraunhofer approximation may be used for $\psi_{n}(x)$ and $\chi_{n}(x)$ too; see Eqs. (3.22) and (5.15). Then, supposing $\kappa / m \ll 1$ after a little algebra, we arrive at the expressions

$\left|a_{n}\right|^{2} \underset{x \kappa \gg 1}{\longrightarrow} \frac{1}{(m+1)^{2}}+\frac{m-1}{m+1} \cos ^{2}\left(x-\frac{n \pi}{2}\right)+O\left(\frac{\kappa}{m}\right)$,

$\left|b_{n}\right|^{2} \underset{x \kappa \gg 1}{\longrightarrow} \frac{1}{(m+1)^{2}}+\frac{m-1}{m+1} \sin ^{2}\left(x-\frac{n \pi}{2}\right)+O\left(\frac{\kappa}{m}\right)$,

while $\left|a_{n}^{(\mathrm{PEC})}\right|^{2}$ and $\left|b_{n}^{(\mathrm{PEC})}\right|^{2}$ in the same approximation equal $\cos ^{2}\left(x-\frac{n \pi}{2}\right)$ and $\sin ^{2}\left(x-\frac{n \pi}{2}\right)$, respectively; see Eqs. (3.19), (3.22), (3.26), and (5.15). Thus, the obtained asymptotic profiles are different from those for $\left|a_{n}^{(\mathrm{PEC})}\right|^{2}$ and $\left|b_{n}^{\text {(PEC) }}\right|^{2}$, indeed. This solves the puzzle.

We should emphasize that the applicability conditions for Eqs. (8.2) and (8.3) are

$$
x \gg 1, \quad \kappa x \gg 1, \quad m \gg \kappa ;
$$

i.e., in contrast to most results of the present paper, they do not imply $m$ necessarily to be large.

It is important to stress that, though in the leading approximation the obtained asymptotical expressions for $\left|a_{n}\right|^{2}$ and $\left|b_{n}\right|^{2}$ are $\kappa$-independent, the asymptotics themselves begin at $x \sim 1 / \kappa$; see Eq. (8.1). At $\kappa \rightarrow 0$ the asymptotics shift to infinity and disappear. Such a behavior makes the case of any large $x$ and small but finite $\kappa$ qualitatively different from the nondissipative limit, discussed above and in various previous publications; see, e.g., [36,46].

\section{B. Large lossy sphere: The lower bounds for the scattering cross section and the upper bound for the absorption one}

Equations (8.2) and (8.3) result in an important and rather unexpected conclusion. Let us consider the partial scattering efficiency $Q_{\text {sca, } n}$ (the corresponding partial cross section normalized over the geometric cross section of the sphere $\pi R^{2}$ ). In terms of the scattering coefficients this quantity is expressed as follows [36]:

$$
Q_{\mathrm{sca}, n}=\frac{2(2 n+1)}{x^{2}}\left\{\left|a_{n}\right|^{2}+\left|b_{n}\right|^{2}\right\} .
$$

At $x \kappa \gg 1, \kappa \ll m$, in accord with Eqs. (8.2) and (8.3), in the leading in $\kappa / m$ approximation Eq. (8.5) reads

$$
Q_{\mathrm{sca}, n} \cong \frac{2(2 n+1)}{x^{2}} \frac{m^{2}+1}{(m+1)^{2}} .
$$

Note that in this case the corresponding dimensional partial cross section $\sigma_{\text {sca, } n}=\pi R^{2} Q_{\text {sca, } n}$ occurs independent of both $\kappa$ and $R$, while its $n$ dependence is reduced to that in the prefactor of general expression Eq. (8.5). This is true as long as the Fraunhofer regime is valid. At a fixed value of $x \gg 1$ it corresponds to $n \leqslant N \equiv[x / 2]$, where [x/2] stands for the integer part of $x / 2$; see the comment to Eq. (3.22).

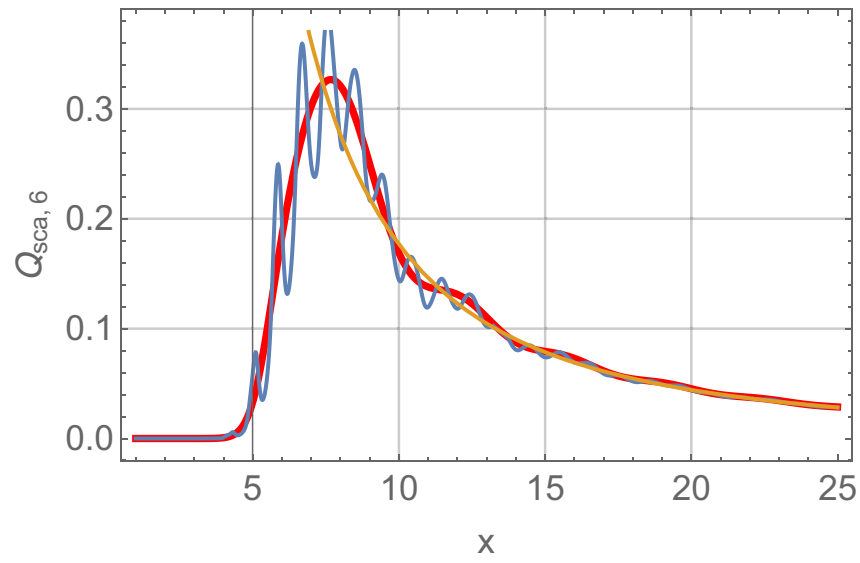

FIG. 10. Partial scattering efficiency at $n=6$ as a function of the size parameter. The real part of the refractive index is fixed for all the curves at $m=4$. The imaginary part equals 0.1 (thin blue oscillating curve) and 1.0 (thick red curve). The thin yellow curve shows asymptotic dependence Eq. (8.6). The Fraunhofer regimes corresponds to $x \geqslant 12$. Note that in this regime all the three curves are very close to each other.

The accuracy of Eq. (8.6) is very good. As an example, dependencies $Q_{\text {sca,6 }}$ on $x$ at $m=4$ and two values of $\kappa(0.1$ and 1.0), as well as the one given by Eq. (8.6), are shown in Fig. 10. For $n=6$ the Fraunhofer regimes is valid at $x \geqslant 12$. At $x=12$ all the three curves are already very close to each other. Then, at further increase of $x$ they practically merge.

Equation (8.6) makes it possible to readily calculate the net contribution of the modes obeying this asymptotic $\left(Q_{\text {sca }}^{\text {(Frhf })}\right)$ to the overall scattering efficiency $\left(Q_{\text {sca }}\right)$ :

$$
Q_{\text {sca }}^{\text {(Frhf })}=\sum_{n=1}^{N} \frac{2(2 n+1)}{x^{2}} \frac{m^{2}+1}{(m+1)^{2}}=\frac{2 N(N+2)}{x^{2}} \frac{m^{2}+1}{(m+1)^{2}} .
$$

If here $N=[x / 2] \gg 1$, expression Eq. (8.7) is reduced to a simple $x$-independent form,

$$
Q_{\text {sca }}^{(\text {Frhf })} \underset{x \gg 1}{\longrightarrow} \frac{m^{2}+1}{2(m+1)^{2}} \underset{m \gg 1}{\longrightarrow} \frac{1}{2} .
$$

Of course, at large $x$ there is a number of the modes with $n<$ $x / 2$, which do not belong to the Fraunhofer range but still make a substantial contribution to the overall scattering efficiency. Since any partial efficiency, by definition, is a positive quantity [see Eq. (8.5)], it means that $Q_{\mathrm{sca}}^{(\mathrm{Frhf})}$ should be regarded as the lower bound for $Q_{\text {sca }}$.

Recall now the well-known facts that the extinction efficiency for a sphere, $Q_{\text {ext }}$, appears to approach the limiting value 2 as the size parameter increases (the extinction paradox) [36] and that the absorption efficiency, $Q_{\text {abs }}$, equals $Q_{\text {ext }}-Q_{\text {sca }}$. Then Eq. (8.8) yields the upper bound for $Q_{\mathrm{abs}}$ :

$$
Q_{\mathrm{abs}}<3 / 2 \text { at } x \gg 1, \quad x \kappa \gg 1, \quad \kappa \ll m .
$$

Thus, we have obtained that under the specified conditions the scattering cross section of a large lossy sphere cannot be smaller than half of its geometric cross section, while its absorption cross section cannot exceed three halves of the geometric one. 


\section{Field within the particle: Individual modes}

As always, we begin the analysis with inspection of the electric modes. The results obtained in Sec. VII show that the key function determining the behavior of the resonances at large variations of $x$ is $B_{n}^{(d)}(x)=\left|\xi_{n}^{\prime}(x)\right|^{2}$. The known expansions of the Bessel functions in powers of their small argument yield the following behavior of $B_{n}^{(d)}(x)$ at $x \rightarrow 0$ :

$$
B_{n}^{(d)}(x) \cong \frac{n^{2} 4^{n} \Gamma^{2}\left(n+\frac{1}{2}\right)}{\pi x^{2(n+1)}},
$$

where $\Gamma\left(n+\frac{1}{2}\right)$ stands for the Gamma function. An increase in $x$ is supplemented with a monotonic decrease in $B_{n}^{(d)}$ until this function reaches its minimal value at $x \approx n$. A further increase in $x$ results in a slow monotonic growth of $B_{n}^{(d)}$, asymptotically approaching unity at $x \rightarrow \infty$; see Eqs. (3.22) and (5.15). Accordingly, the entire domain $0 \leqslant x<\infty$ of variations of $x$ is partitioned into two subdomains: $0<x \leqslant n$, where a sharp fall of $B_{n}^{(d)}(x)$ from infinity to its minimal value below unity occurs, and the one of a slow asymptotical growth $B_{n}^{(d)}(x)$ to unity $(n<x<\infty)$. As an example, functions $B_{n}^{(d)}(x)$ for the first five multipoles are presented in Fig. 11(a).

Thus, at any $n$ both $B_{n}^{(d)}(x)$ and $x B_{n}^{(d)}(x)$ are singular functions at $x \rightarrow 0$. It brings about a dramatic enhancement of the dissipative effects at small $x$; see Eq. (7.4). On the other hand, an increase in $B_{n}^{(d)}(x)$ increases the numerator in the right-hand side of Eq. (7.5), defining the amplitude of the resonance. That is to say, at small $x$ there is a competition between the growth of the amplitude of the resonance owing to the increase in $B_{n}^{(d)}(x)$ in the numerator of Eq. (7.5) and its suppression because of the growth of the same quantity in the denominator of the same equation. In this case we have to distinguish two limits.

(i) Despite the large value of $B_{n}^{(d)}$, the first (smallest) resonant $x_{n, 1}^{\text {(res) }}<n$ still corresponds to weak dissipation $\left[\kappa m x_{n, 1}^{(\text {res })} B_{n}^{(d)}(x) \ll 1\right]$. Then the amplitude of this resonance is the largest for the given $n$. An increase in $x$ gives rise to the fall of the amplitudes of the sequential resonances, first due to the decrease of $B_{n}^{(d)}(x)$ in the numerator of Eq. (7.5) and then owing to the transition to the dissipation-controlled region because of the increase of $\kappa B_{n}^{(d)}(x) m x$ in the denominator of this expression.

(ii) $x_{n, 1}^{\text {(res) }}<n$ is so small [i.e., $B_{n}^{(d)}\left(x_{n, 1}^{\text {(res) }}\right)$ is so large], that $\kappa B_{n}^{(d)}\left(x_{n, 1}^{(\text {res })}\right) m x_{n, 1}^{(\text {res })} \gg 1$, despite smallness of $\kappa$. Then, the amplitude of the corresponding resonance approximately equals

$$
\operatorname{Max}\left\{\left|d_{n}\right|^{2}\right\} \cong \frac{1}{\left[\psi_{n}^{\prime}\left(m x^{(\mathrm{res})}\right) \kappa x^{(\mathrm{res})}\right]^{2} B_{n}^{(d)}\left(x^{(\mathrm{res})}\right)} ;
$$

see Eq. (7.5). Since, by definition, in a given cascade $x_{n, 1}^{\text {(res) }}<$ $x_{n, 2}^{\text {(res) }}<x_{n, 3}^{\text {(res) }}<\cdots$, the next resonances in the same cascade result in a decrease of $B_{n}^{(d)}\left(x_{n, p}^{(\text {res })}\right)$ and, hence, in an increase of their amplitude. It goes on in this manner until the decease of $B_{n}^{(d)}$, eventually, drives the problem out of the dissipationcontrolled regime. Then, a further increase in $x^{(\mathrm{res})}$ results in the effects, described above in item (i).

Thus, now we have two dissipation-controlled domains: the first at small $x$ and the second at large, separated by a nondissipative domain. The maximal amplitude of the resonance mode is achieved at $x^{(\text {res })}$ situated at the boundary between the first dissipation-control domain the nondissipative one.

It is important to stress also the quite different asymptotic behavior of profiles $\left|d_{n}(m)\right|^{2}$ at a fixed $x$, and $\left|d_{n}(x)\right|^{2}$ at a fixed $m$, respectively. If $\left|d_{n}(m)\right|^{2}$ at $m \rightarrow \infty$ converges to a certain universal periodic function [see Eq. (7.7)], $\left|d_{n}(m)\right|^{2}$ at $x \rightarrow \infty$ vanishes as $1 /(\kappa x)^{2}$, owing to Eq. (7.5) and limits

$$
\left|\psi_{n}^{\prime}\left(m x^{(\mathrm{res})}\right)\right| \underset{x^{(\mathrm{res})} \rightarrow \infty}{\longrightarrow} 1, \quad B_{n}^{(d)}(x) \underset{x \rightarrow \infty}{\longrightarrow} 1 .
$$

The behavior of $\left|c_{n}\right|^{2}$, in general, is analogous to that discussed above for $\left|d_{n}\right|$. However, in contrast to $B_{n}^{(d)}(x)$, function $B_{n}^{(c)}(x)$ is monotonically decreasing; see Fig. 11(b). This difference in $B_{n}$ results in a certain difference in the shape of the envelopes of the resonances for $\left|d_{n}(x)\right|$ and $\left|c_{n}(x)\right|$; see Fig. 12.

\section{Field within the particle: Overlap of resonances}

One of the key features of the problem in question is the overlap of a large number of resonances, which may result in a creation within the particle "bright spots" with a giant concentration of the electromagnetic field. The corresponding field structure is determined by the coordinate dependence of the resonant modes. Its detailed inspection requires a separate consideration and will be reported elsewhere. In the present paper we discuss just the necessary conditions for such bright spots to come into being, namely the overlap of profiles of the modula of the scattering coefficients, describing the field within the particle. As usual, for the sake of briefness we restrict the discussion with the properties of $\left|d_{n}\right|^{2}$ solely. The behavior of $\left|c_{n}\right|^{2}$ may be studied in the same manner and exhibits similar peculiarities.

Overlap of two resonances means that the mismatch between the positions of the maxima of their lines is smaller than the largest linewidth. Therefore, to begin with, we have to discuss the linewidths of the resonances at a fixed $m$ and varying $x$. To this end, according to the stipulated-above general rules, we have to replace in Eq. (7.2) $x \delta m \rightarrow m \delta x, m^{(\mathrm{res})} \rightarrow m$, and $x \rightarrow x^{(\text {res })}$. This brings about the following expression for the linewidth:

$$
\gamma_{\left|d_{n}\right|^{2}}^{(\kappa, x)}=2 \frac{1+\kappa B_{n}^{(d)}\left(x^{(\mathrm{res})}\right) m x^{(\mathrm{res})}}{B_{n}^{(d)}\left(x^{(\mathrm{res})}\right) m^{2}} .
$$

Thus, in the nondissipative region $\left[\kappa B_{n}^{(d)}(x) m x \ll 1\right]$ the linewidth is $2 /\left[B_{n}^{(d)}(x) m^{2}\right]$, while in the dissipation-controlled region $\left[\kappa B_{n}^{(d)}(x) m x \gg 1\right]$ it is $2 \kappa x / m$.

On the other hand, the resonant values of $x$ are defined by the condition $\psi_{n}\left(m x^{\text {(res, } E)}\right)=0$ for the electric modes and $\psi_{n}^{\prime}\left(m x^{(\mathrm{res}, H)}\right)=0$ for magnetic; see Eqs. (3.21) and (3.29). At relatively small $x$, lying below the Fraunhofer regime, different solutions of these equations are situated at distances of the order of $1 / \mathrm{m}$. It is always much larger than the linewidth in the nondissipative region and still larger than that in the dissipation-controlled case, provided that $x$ is of the order of unity or smaller. Therefore, the overlap of the resonances in these regions may occur just accidentally.

However, in the Fraunhofer regime, the situation is changed drastically. In this regime points of different resonances are 

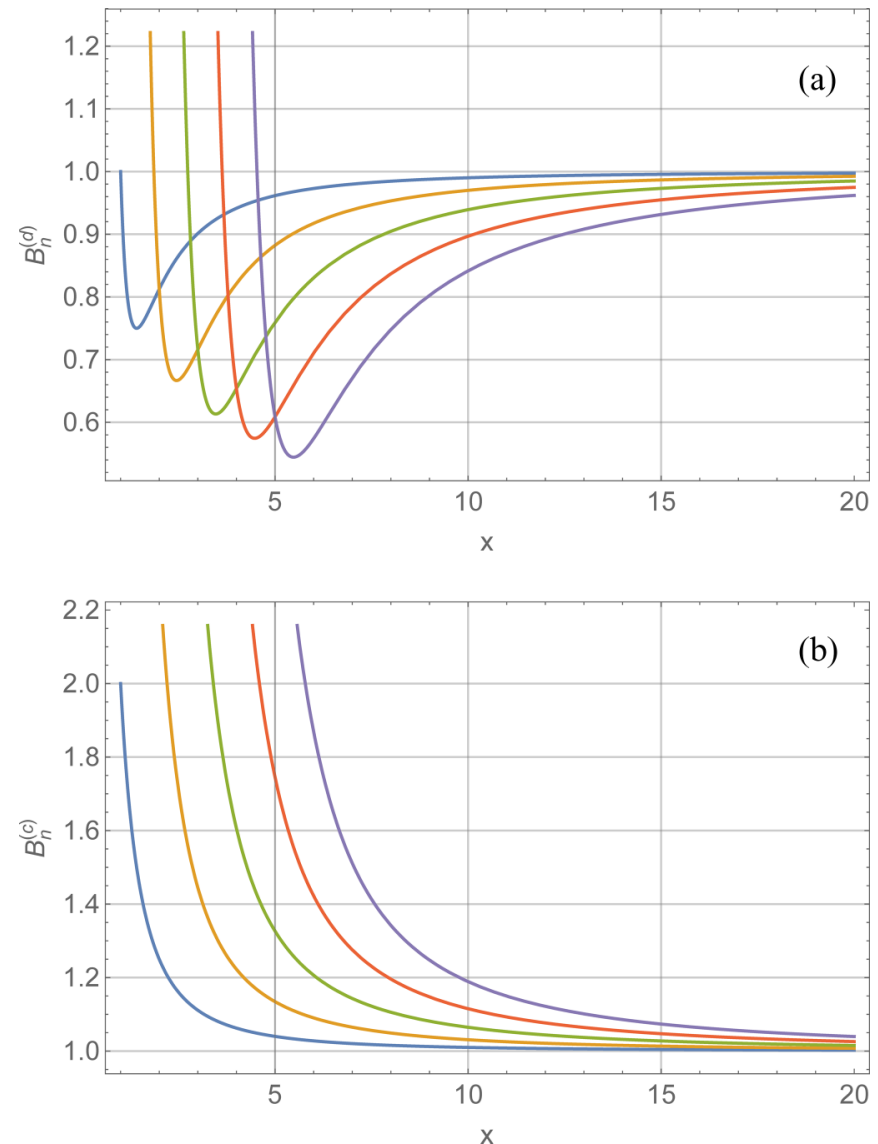

FIG. 11. Behavior of $B_{n}^{(d)}(x) \equiv\left|\xi_{n}^{\prime}(x)\right|^{2}$ and $B_{n}^{(c)}(x) \equiv\left|\xi_{n}(x)\right|^{2}$ for the first five multipoles. Curves from left to right correspond to $n=1,2,3,4$, and 5, respectively.

going to merge. In the leading (in $1 / m x$ ) approximation they just coincide; see Sec. III. To resolve the mismatch between the points of different resonances, we have to go beyond the leading approximation.

The increase of the accuracy forces us to impose more strict applicability conditions for the validity of the approximation. Therefore, in this section we suppose that condition $z>2 n$ employed in the preceding sections is replaced with $z \gg n^{2}$; see the comment below Eq. (3.22).

Utilizing the asymptotic expansion of the Riccati-Bessel functions at large values of their argument [42] and taking into account the first subleading terms, instead of Eq. (3.23) we arrive at the following equation, determining the points of the electric mode resonances,

$$
\sin \left(\rho_{E}-\frac{n \pi}{2}\right)+\frac{n(n+1)}{2 \rho_{E}} \cos \left(\rho_{E}-\frac{n \pi}{2}\right) \cong 0,
$$

where $\rho_{E}>n^{2}$ stands for $m x^{(\text {res, } E)}$. Looking for a solution of this equation in the form $\rho_{E}=\rho_{E}^{(0)}+\delta \rho_{E} ;\left|\delta \rho_{E}\right| \ll 1$, where $\rho_{E}^{(0)}=[(2 p+n) \pi / 2]$, it is easy to obtain that

$$
\delta \rho_{E} \cong-\frac{n(n+1)}{\pi(n+2 p)} .
$$

The solution is valid at $n(n+1) \ll \pi(n+2 p)$. The corresponding mismatch between different resonances is $\delta \rho_{E} / \mathrm{m}$.
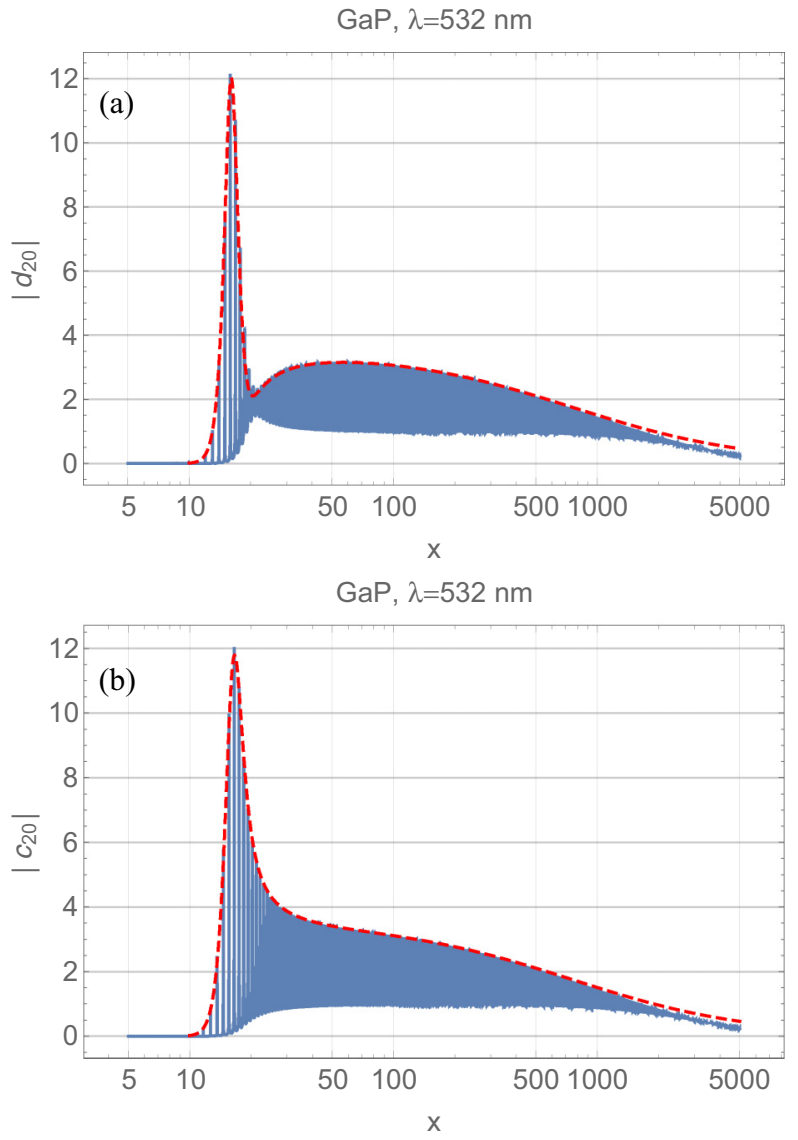

FIG. 12. Typical behavior of $\left|d_{n}(x)\right|$ and $\left|c_{n}(x)\right|$ at $n=20$ (as an example) for a particle made of $\mathrm{GaP}$. Blue solid lines correspond to the exact solution [Eqs. (3.6) and (3.9)]; the envelopes, calculated according to the approximate Eqs. (7.5) and (7.8) are shown as red dashed lines. Note strongly nonmonotonic dependence of the amplitude of the resonant oscillations on $x$ due to the enhancement of the dissipative effects at certain domains of variations of $x$ and a "bottle neck" in the dependence $\left|d_{n}(x)\right|$ at the proximity of $x=n=20$. The bottleneck is originated in the corresponding minimum in $B_{n}^{(d)}(x)$, which does not have $B_{n}^{(c)}(x)$; cf. the envelopes with the profiles of $B_{n}^{(d, c)}$ in Fig. 11. See the text for details.

Strictly speaking, we have to add to this mismatch another one, caused by the departure of the position of the maxima of $\left|d_{n}\right|^{2}$ from the resonance points, determined according to the employed condition $\psi_{n}(m x)=0$. However, according to Eq. (7.2) this mismatch equals $A_{n}^{(d)}(x) / m^{2}$ [we recall that to apply Eq. (7.2) to our case we have to replace $x \delta m \rightarrow m \delta x]$. At large $m$ this quantity is small relative to $\delta \rho_{E} / m$ and may be neglected.

Similar inspection of $\left|c_{n}\right|^{2}$ gives rise to $\rho_{H}=\rho_{H}^{(0)}+\delta \rho_{H}$, with

$$
\rho_{H}^{(0)}=\frac{(n+2 p+1) \pi}{2}, \quad \delta \rho_{H} \cong \frac{n(n+1)}{\pi(n+2 p+1)} .
$$

Let us put it all together. According to the results discussed in Sec. III, there are two types of possible overlaps. The first corresponds to the overlap of the modes of the same type (e.g., electric-electric, or magnetic-magnetic) with different $n$ and $p$, changed in such a manner that the sum $n+2 p$ remains fixed. 
The adjacent candidates for this overlap have the difference in $n$ equals 2 and the difference in $p$ equals 1 . According to Eqs. (8.13) and (8.14) the mismatches between the resonance points for these two modes are

$$
\left(\Delta x_{E}\right)_{n, n+2} \cong\left(\Delta x_{H}\right)_{n, n+2} \cong \frac{2(3+2 n)}{m \pi N},
$$

where integer $N=n_{E}+2 p_{E}=n_{H}+2 p_{H}+1$ satisfies the condition $\pi N / 2=\rho_{E}^{(0)}=\rho_{H}^{(0)}=m x^{(\text {res })}$.

The candidates for the second type of the overlap are modes with the different nature: electric-magnetic. In this case the adjacent modes have both $n$ and $p$ differed in unity; however, the signs of these mismatches are opposite [see Eqs. (8.13) and (8.14)]. Then the corresponding mismatch is

$$
\left(\Delta x_{E, H}\right)_{n, n+1} \cong \frac{2(n+1)^{2}}{m \pi N} .
$$

In all the cases the mismatches monotonically increase with an increase in $n$. A rough estimate of the number of modes, which may overlap, yields from the applicability condition of the Fraunhofer regime: $\rho>n^{2}$. For a given $x^{(\mathrm{res})}$ it results in the inequality $n<\sqrt{N} \sim \sqrt{m x^{(\text {res })}}$.

Thus, at $n \gg 1$ the mismatches between the modes of the same type are scaled as $n /(m N) \sim n /\left(m^{2} x\right)$, while the ones between the modes of different types are scaled as $n^{2} /(m N) \sim n^{2} /\left(m^{2} x\right)$. On the other hand, the linewidth in the dissipation-controlled region is scaled as $\kappa x / m$; see Eq. (8.11). An increase in $x$ decreases $\Delta x$ and increases the linewidth. The overlap begins when the latter becomes larger than the former. First, it happens with the modes of the same type at $x=x_{\mathrm{ovlp}}$, where

$$
x_{\mathrm{ovlp}} \sim \sqrt{\frac{n}{m \kappa}} .
$$

A further growth of $x$ increases the number of the overlapping modes until all modes lying in the Fraunhofer range overlap. It happens at $x>X_{\text {ovlp }}$, where $X_{\text {ovlp }}$ is such a value of $x$, when $(\Delta x)_{1, \sqrt{N}}$ becomes equal to or smaller than the linewidth. Here $(\Delta x)_{1, \sqrt{N}}$ stands for the mismatch between the mode with $n=1$ and the mode with the maximal $n$ still belonging to the Fraunhofer range, i.e., with $n \sim \sqrt{N}$. According to Eqs. (8.13) and (8.14), $(\Delta x)_{1, \sqrt{N}} \sim\left(n^{2} / m N\right)_{n=\sqrt{N}}=1 / m$. It yields a simple estimate $X_{\text {ovlp }} \sim 1 / \kappa$.

As it follows from Eq. (7.5), $\operatorname{Max}\left\{\left|d_{n}\left(X_{\text {ovlp }}\right)\right|\right\} \sim 1$. Then, at the points where all the overlapping modes are summarized coherently with the weight factor of the order of unity, the resulting amplitude of the electric (magnetic) field is $\sqrt{N}$ times greater (the density of energy $N$ times greater) than the one in the incident wave.

\section{E. Examples of the discussed effects for a sphere of $\mathrm{GaP}$ at $\lambda=532 \mathrm{~nm}$}

To give a certain impression about a manifestation of the discussed resonances in a possible real experiment we produce some results for a particle made of gallium phosphide. The corresponding plots are presented in Fig. 13.

Our calculations for GaP show also that the absolute maximum for a single resonant mode is achieved for the first resonance of mode $c_{5}$ at $x \approx 2.318$. The corresponding value of $\left|c_{5}\right|$ equals 34.641. The first resonant of $\left|d_{5}\right|$ gives rise

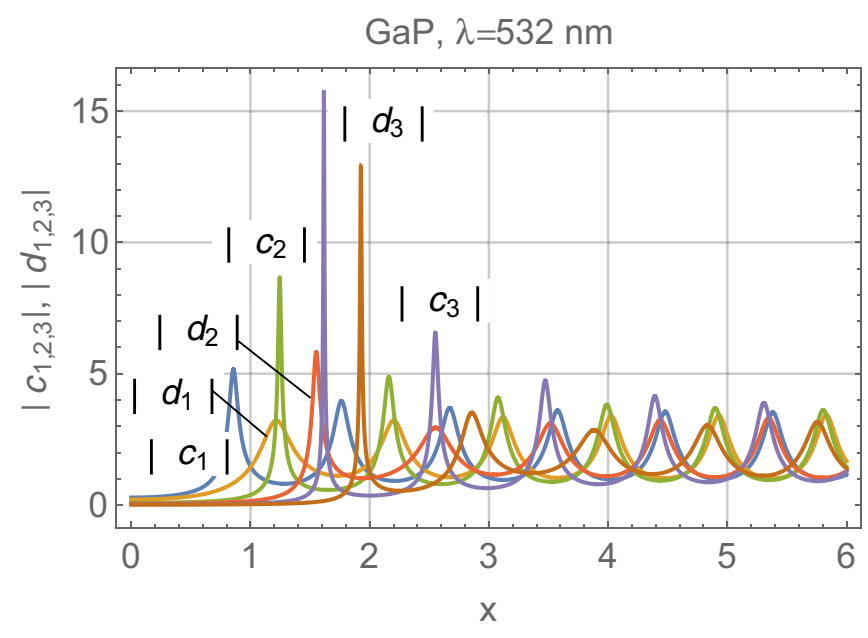

FIG. 13. Cascades of resonances for $\left|c_{n}\right|$ and $\left|d_{n}\right|$ at a fixed refractive index and varying size parameter, $x$, for a spherical particle made of GaP, irradiated in a vacuum by a plane linearly polarized electromagnetic wave with $\lambda=532 \mathrm{~nm}$. Calculations are according to Eqs. (3.6) and (3.9). Gradual overlap of resonances for $\left|c_{1}\right|-\left|d_{2}\right|-\left|c_{3}\right|$ and $\left|d_{1}\right|-\left|c_{2}\right|-\left|d_{3}\right|$ at an increase in $x$ is seen clearly. Note the broadening of the resonance lines with an increase in $x$ and an increase of the amplitudes of the first resonances in each cascade with an increase in $n$. The first resonances in each cascades have the largest amplitudes. The large mismatches between the first resonances in the cascades for $\left|c_{1}\right|-\left|d_{2}\right|$ and $\left|c_{2}\right|-\left|d_{3}\right|$ are generic, while the overlaps of the first resonances in the cascades for $\left|d_{1}\right|-\left|c_{2}\right|$ and $\left|d_{2}\right|-\left|c_{3}\right|$ are accidental. For more details, see the text.

to the close value: 33.161 at $x \approx 2.626$. The density of the electromagnetic energy in the bright spots for these solitary resonances is about $10^{3}$ greater than that for the incident wave. Meanwhile, multiple overlap of not-so-sharp resonances for a particle with $x \approx 25$ may produce bright spots with the density of energy $10^{4}$ relative to that in the incident wave.

As an example, the field distributions for a spherical GaP particle at $\lambda=532 \mathrm{~nm}$ are presented in Fig. 14 for two typical cases: The discussed above sharp individual magnetic resonance at $x=2.318\left(c_{5}\right)$ and a multiple overlap of resonant modes at $x=5.380$ (see Fig. 13). The electric and magnetic fields are normalized over the amplitude of the electric field in the incident wave. These field distributions reveal several interesting features, namely the following.

(i) The overall volume of the bright spots at the case of the individual resonance is larger than that in the case of the multiple overlap of the resonances. However, if in the former case the bright spots have rather sharp boundaries, in the latter the boundaries are much broader (the light blue areas occupy the substantial part of the particle). In other words, at the individual resonance the regions of the extreme field concentration are separated with "dark" region with low field intensity. At the multiple overlap of the resonances the concentration of the field may be weaker, but it occurs in a considerable part of the particle.

(ii) The maximum at the center of the sphere corresponds to either electric $\left(d_{1}\right)$ or magnetic $\left(c_{1}\right)$ dipole mode resonances, since the field distributions for all other multipoles vanish at this point. In contrast, the secondary maxima along radial direction indicate the excitation of higher-order modes in the 

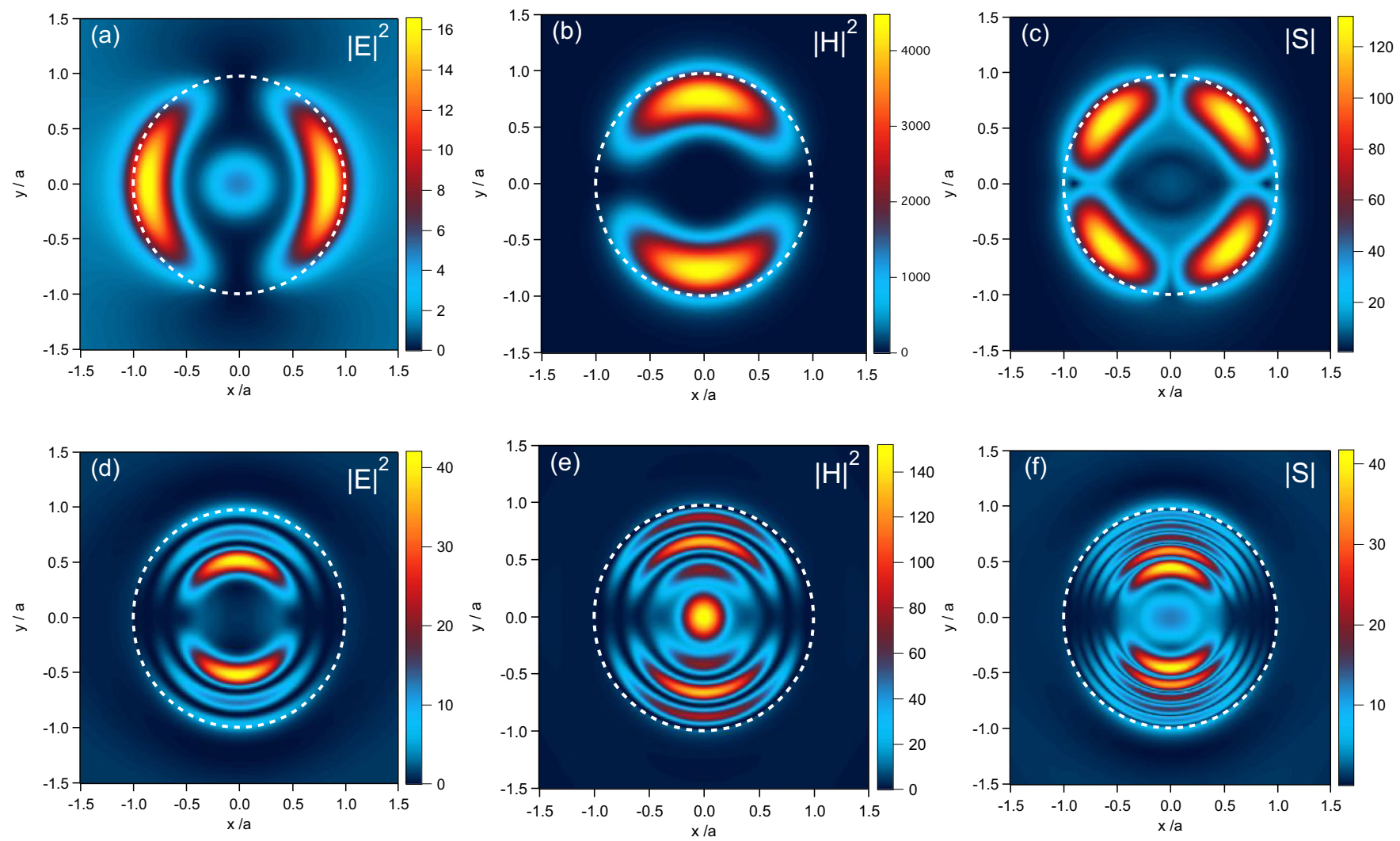

FIG. 14. Distribution of electric $|E|^{2}$, magnetic $|H|^{2}$ fields, and the Poynting vector $|S|$ within a GaP sphere, irradiated in a vacuum by a plane linearly polarized electromagnetic wave with $\lambda=532 \mathrm{~nm}$. Calculations are according to the exact Mie solution. The wave propagates along $\mathbf{Z}$ axis with vector $\mathbf{E}$ directed along the $\mathbf{X}$ axis (do not confuse this $\mathbf{X}$ with the size parameter $x$ ). The electric and magnetic fields are normalized over the amplitude of the electric field of the incident wave. The fields are presented in the plane $z=0$. The boundary of the sphere is shown as white dashed line. The size parameter for panels (a)-(c) equals 2.318; for (d)-(f) it is 5.380. See the text for details.

overlapping regime. It is interesting to note that, in contrast to plasmonic particles, where the absolute maximum of the fields is achieved at their surfaces, presently the absolute maximum is close to, or just at the center of the particle (the dominant role of the dipole modes).

(iii) Though the maximum values of the magnetic field at the centers of the bright spots for the individual magnetic resonance [Fig. 14(b)] is considerably larger than that at the overlap of the resonances [Fig. 14(e)], the case for the electric field is opposite; see Figs. 14(a) and 14(d), respectively.

Feature (i) makes it possible to employ the particle as an effective optical nanocavity with both narrow (individual resonance) and broad (overlap of resonances) lines. Features (ii) and (iii) provide plenty of degrees of freedom to control and engineer a desired field distribution. All this may be very important for numerous applications. However, as said already, a detailed inspection of the field structure lies beyond the scope of the present paper and will be reported elsewhere.

\section{CONCLUSIONS}

Concluding this extended discussion, we may say that the detailed study of the light scattering by a high-refractive-index particle with low dissipation discussed in the present paper has revealed a number of new important features of the problem. In particular, we have shown that while at increasing $m$ the partial scattered waves outside the particle for any fixed values of $x$ and $n$ tend to the limits, corresponding to the light scattering by a perfectly reflective sphere, the field within the particle does not have any limit at all.

The reason for this difference in the behavior between the solutions of the outer and inner problems is related to the different manifestations of the infinite sequences of the cascades of the Mie resonances in the two problems. Specifically, for the scattered field outside the particle each Mie resonance in a cascade has the asymmetric Fano line shape. As $m$ increases the resonances are suppressed, and the expressions for the scattering coefficients converge to the corresponding $m$-independent quantities for the perfectly reflecting, PEC sphere.

For the field within the particle, each individual resonance exhibits the Lorentzian line shape. In this case, while at large $m$ the profile at a fixed distance from its bottom converges to a certain universal form, the peak values of the modulus of the electric (magnetic) field amplitudes increase with an increas in $m$. Thus, the increase in $m$ makes the resonances more pronounced. At finite dissipation the growth of the amplitudes of the resonance eventually saturate, and the resonance lines become the periodic functions of $m$.

It is important that at large-enough $m$ the positions of the electric resonances for a partial mode with multipolarity $n$ correspond to those for magnetic resonances with multipolarity 
$n+1$, the electric mode with multipolarity $n+2$, etc. The same is true for the magnetic modes.

For a given multipolarity the points of both types of the resonances together (i.e., electric and magnetic) are situated in the $m$ axis periodically with the period equal to the half of the period for each type of the resonances separately. It means the points of the magnetic resonances are located just in the middle of the distance between the adjacent points of the electric resonances and vice versa.

We have shown that the series of the Fano resonances for HRI particles is a generic, intrinsic feature of the lightscattering problem, which can be generalized to an arbitrary diffraction problem in wave phenomena (e.g., scattering of sound). In these problems the field scattered by the same scatterer, but with perfectly reflecting properties, plays the role of a background partition, while the modes resonantly excited in the scatterer correspond to the resonant ones.

The characteristics of the Fano profiles, including a simple expression for the asymmetry parameter $q$ [see Eqs. (5.4) and (5.7)], have been obtained from "the first principles" based upon the identical transformations of the exact Mie solution. It has made possible to obtained the rigorous generalization of the Fano resonances to the case with finite dissipation [see Eqs. (7.9)] and to discuss the degradation of the Fano profile at an increase in the dissipation; see Fig. 8 as well as the corresponding comment in Sec. VII B.

We have demonstrated that in the nondissipative limit the discussed resonances (for both the inner and outer problems) possess a scale invariance, so that at any fixed value of the size parameter $x$ any resonance line in the infinite cascades of the resonances may be reduced to the universal, $m^{(\text {res })}$-independent form by the scale transformations, Eqs. (6.1), (6.2). It should be stressed that the universality extends to both the shape of the lines and the mutual positions of the lines with respect to each other for modes with different multipolarity and/or of different types (electric or magnetic). The quantitative applicability conditions for the nondissipative and dissipation-controlled regimes as well as the corresponding crossover points have been obtained in the explicit form.

Under very general conditions [Eq. (8.4)] the scattering cross section of a large lossy sphere cannot be smaller than half of its geometric cross section, while its absorption cross section cannot exceed three halves of the geometric one. It is important that for the validity of these results the refractive index of the sphere not necessarily should be large, provided conditions in Eq. (8.4) hold.

Regarding the peculiarities of the resonances at a fixed refractive index and a varying size parameter $x$, we have shown that, generally speaking, the entire domain $0 \leqslant x<\infty$ is partitioned into three subdomains: Two dissipation-controlled ones (at small and very large $x$ ) are separated by a nondissipative subdomain. The explicit expressions, determining the boundaries between the subdomains as well as the formulas for the line shape and linewidth, have been derived. The linewidth is minimal in the nondissipative domain and increases in the dissipation-controlled subdomains with an increase in the departure of $x$ from the corresponding boundary.

For the size parameter lying in the nondissipative subdomain a high concentration of the electromagnetic field within the particle may be achieved owing to the individual narrow-line partial resonances of modes with a high $Q$ factor. In this case the field structure within the particle has a high contrast. That is to say, the boundaries between bright spots with a high concentration of the field and dark regions with weak field are sharp; see Figs. 14(a)-14(c). For the realistic values of the complex refractive index, typical for a number of common semiconductors in the visible and near-IR range of the spectrum, the peak value of the density of electromagnetic energy in the bright spots in this case may exceed the one in the incident plane wave by three orders of magnitude and even more than that.

For the size parameter, lying in the dissipation-controlled subdomains, a high concentration of the field may be realized due to multiple overlap of rather broad resonance lines of modes of different types (magnetic and electric) and different multipolarity. In this case the contrast of the field structure is much lower than the one at the single-mode resonance; $c f$. Figs. 14(d)-14(f) and 14(a)-14(c).

The discussed effect of the giant concentration of the electromagnetic field within a particle with a high refractive index together with the highlighted differences between the two cases (the individual resonance and the multiple overlap of the resonances) provide plenty of opportunities to design and engineer new materials and/or nanodevices with unique properties. They may be utilized in numerous applications in medicine (such as, e.g., cancer therapy [47]), in biology (new sensors and markers), nanoelectronics and nonoptics (fabrication of high-nonlinear nanostructures), telecommunication, data storage and processing, etc. We hope that our paper may stimulate further progress in these important fields.

\section{ACKNOWLEDGMENTS}

The authors are grateful to Y. Kivshar and B. S. Luk'yanchuk for the critical reading of the manuscript and valuable comments. The work of AEM was supported by the Australian Research Council via Future Fellowship program (Grant No. FT110100037).

\section{APPENDIX: PROOF OF IDENTITY, EQ. (4.11)}

The proof is based upon Eq. (3.7). According to it and the presentation of $\xi_{n}$ in the form $\xi_{n}=\psi_{n}-i \chi_{n}$, with real functions $\psi_{n}$ and $\chi_{n}$, we may write a chain of identities,

$$
\begin{aligned}
\frac{\xi_{n}(x)}{\xi_{n}^{\prime}(x)} & \equiv \frac{\psi_{n}^{\prime}(x) \xi_{n}(x)}{\psi_{n}^{\prime}(x) \xi_{n}^{\prime}(x)} \equiv \frac{\psi_{n}(x) \xi_{n}^{\prime}(x)-i}{\psi_{n}^{\prime}(x) \xi_{n}^{\prime}(x)} \\
& \equiv \frac{\psi_{n}(x)}{\psi_{n}^{\prime}(x)}-\frac{i \xi_{n}^{\prime *}(x)}{\psi_{n}^{\prime}(x)\left|\xi_{n}^{\prime}(x)\right|^{2}},
\end{aligned}
$$

where the asterisk means complex conjugation. Then, bearing in mind the same presentation $\xi_{n}=\psi_{n}-i \chi_{n}$, and taking the imaginary part of Eq. (A1) we obtain

$$
\operatorname{Im} \frac{\xi_{n}(x)}{\xi_{n}^{\prime}(x)}=-\frac{1}{\left|\xi_{n}^{\prime}(x)\right|^{2}} .
$$

Finally, multiplying it by $\xi_{n}^{\prime 2}(x)$ and taking modulus, we arrive at the identity Eq. (4.11). 
[1] Q. Zhao, J. Zhou, F. Zhang, and D. Lippens, Mie resonancebased dielectric metamaterials, Mater. Today (Oxford, UK) 12, 60 (2009).

[2] M. V. Rybin, A. B. Khanikaev, M. Inoue, K. B. Samusev, M. J. Steel, G. Yushin, and M. F. Limonov, Fano Resonance between Mie and Bragg Scattering in Photonic Crystals, Phys. Rev. Lett. 103, 023901 (2009).

[3] A. B. Evlyukhin, C. Reinhardt, and B. N. Chichkov, Multipole light scattering by nonspherical nanoparticles in the discrete dipole approximation, Phys. Rev. B 84, 235429 (2011).

[4] I. Staude, A. E. Miroshnichenko, M. Decker, N. T. Fofang, S. Liu, E. Gonzales, J. Dominguez, T. S. Luk, D. N. Neshev, I. Brener, and Y. Kivshar, Tailoring directional scattering through magnetic and electric resonances in subwavelength silicon nanodisks, ACS Nano 7, 7824 (2013).

[5] I. M. Hancu, A. G. Curto, M. Castro-López, M. Kuttge, and N. F. van Hulst, Multipolar interference for directed light emission, Nano Lett. 14, 166 (2014).

[6] A. I. Kuznetsov, A. E. Miroshnichenko, Y. Hsing Fu, V. Viswanathan, M. Rahmani, V. Valuckas, Z. Ying Pan, Y. Kivshar, D. S. Pickard, and B. Luk'yanchuk, Split-ball resonator as a three-dimensional analog of planar split-rings, Nat. Commun. 5 (2014).

[7] Z. Li, S. Zhang, L. Tong, P. Wang, B. Dong, and H. Xu, Ultrasensitive size-selection of plasmonic nanoparticles by Fano interference optical force, ACS Nano 8, 701 (2014).

[8] H. Chen, S. Liu, J. Zi, and Z. Lin, Fano resonance-induced negative optical scattering force on plasmonic nanoparticles, ACS Nano 9, 1926 (2015).

[9] L. Novotny and B. Hecht, Principles of Nano-optics (Cambridge University Press, New York, 2006).

[10] M. V. Rybin, P. V. Kapitanova, D. S. Filonov, A. P. Slobozhnanyuk, P. A. Belov, Y. S. Kivshar, and M. F. Limonov, Fano resonances in antennas: General control over radiation pattern, Phys. Rev. B 88, 205106 (2013).

[11] V. Klimov, Nanoplasmonics (Pan Stanford, Singapore, 2014).

[12] S. Jahani and Z. Jacob, All-dielectric metamaterials, Nat. Nanotechnol. 11, 23 (2016).

[13] A. B. Evlyukhin, C. Reinhardt, A. Seidel, B. S. Luk'yanchuk, and B. N. Chichkov, Optical response features of Si-nanoparticle arrays, Phys. Rev. B 82, 045404 (2010).

[14] A. I. Kuznetsov, A. E. Miroshnichenko, Y. H. Fu, J. Zhang, and B. Lukyanchuk, Magnetic light, Sci. Rep. 2, 492 (2012).

[15] A. B. Evlyukhin, S. M. Novikov, U. Zywietz, R. L. Eriksen, C. Reinhardt, S. I. Bozhevolnyi, and B. N. Chichkov, Demonstration of magnetic dipole resonances of dielectric nanospheres in the visible region, Nano Lett. 12, 3749 (2012).

[16] D. Landau, L. P. Pitaevskii, and E. Lifshitz, Electrodynamics of Continuous Media, 2nd ed., Course of Theoretical Physics (Butterworth-Heinemann, Oxford, UK, 1984), Vol. 8.

[17] H. C. van de Hulst, Light Scattering by Small Particles (Dover, Mineola, NY, 1981).

[18] M. I. Tribelsky, A. E. Miroshnichenko, and Y. S. Kivshar, Unconventional Fano resonances in light scattering by small particles, Europhys. Lett. 97, 44005 (2012).

[19] J. M. Geffrin, B. García-Cámara, R. Gómez-Medina, P. Albella, L. S. Froufe-Pérez, C. Eyraud, A. Litman, R. Vaillon, F. González, M. Nieto-Vesperinas, J. J. Sáenz, and F. Moreno, Magnetic and electric coherence in forward- and back-scattered electromagnetic waves by a single dielectric subwavelength sphere, Nat. Commun. 3, 1171 (2012).

[20] H. Chen, C. T. Chan, and P. Sheng, Transformation optics and metamaterials, Nat. Mater. 9, 387 (2010).

[21] M. I. Tribelsky and A. E. Miroshnichenko, Giant in-particle field concentration and Fano resonances at light scattering by high-refractive index particles, arXiv:1511.02931.

[22] Z. Ruan and S. Fan, Superscattering of Light from Subwavelength Nanostructures, Phys. Rev. Lett. 105, 013901 (2010).

[23] S. Liu, L. Li, Z. Lin, H. Y. Chen, J. Zi, and C. T. Chan, Graded index photonic hole: Analytical and rigorous full wave solution, Phys. Rev. B 82, 054204 (2010).

[24] M. V. Rybin, K. B. Samusev, I. S. Sinev, G. Semouchkin, E. Semouchkina, Y. S. Kivshar, and M. F. Limonov, Mie scattering as a cascade of Fano resonances, Opt. Express 21, 30107 (2013)

[25] M. V. Rybin, S. I. Sinev, K. B. Samusev, and M. F. Limonov, Cascades of Fano resonances in Mie scattering, Phys. Solid State 56, 580 (2014).

[26] M. V. Rybin, D. S. Filonov, P. A. Belov, Y. S. Kivshar, and M. F. Limonov, Switching from visibility to invisibility via Fano resonances: Theory and experiment, Sci. Rep. 5, 8774 (2015).

[27] U. Fano, Effects of configuration interaction on intensities and phase shifts, Phys. Rev. 124, 1866 (1961).

[28] A. N. Poddubny, M. V. Rybin, M. F. Limonov, and Y. S. Kivshar, Fano interference governs wave transport in disordered systems, Nat. Commun. 3, 914 (2012).

[29] J.-P. Connerade and A. M. Lane, Interacting resonances in atomic spectroscopy, Rep. Prog. Phys 51, 1439 (1988).

[30] K. J. Vahala, Optical microcavities, Nature (London) 424, 839 (2003).

[31] P. Chýlek, Partial-wave resonances and the ripple structure in the Mie normalized extinction cross section, J. Opt. Soc. Am. 66, 285 (1976).

[32] P. Chýlek, J. D. Pendleton, and R. G. Pinnick, Internal and near-surface scattered field of a spherical particle at resonant conditions, Appl. Opt. 24, 3940 (1985).

[33] Z. Wang, Z. Yi, and B. Luk'yanchuk, Near-field focusing of dielectric microspheres: Super-resolution and field-invariant parameter scaling, arXiv:1304.4139.

[34] T. J. Arruda and A. S. Martinez, Electromagnetic energy within magnetic spheres, J. Opt. Soc. Am. A 27, 992 (2010).

[35] M. Born and E. Wolf, Principles of Optics, 7th ed. (Cambridge University Press, Cambridge, UK, 1999).

[36] C. F. Bohren and D. R. Huffman, Absorption and Scattering of Light by Small Particles (Wiley, New York, 1998).

[37] M. Abramowitz and I. A. Stegun, Handbook of Mathematical Functions: With Formulas, Graphs, and Mathematical Tables (Dover, New York, 1965).

[38] C. A. Balanis, Advanced Engineering Electromagnetics, 2nd ed. (Wiley, New York, 2012).

[39] A. Messiah, Quantum Mechanics (North-Holland, Amsterdam, 1964), Vol. 1, p. 490.

[40] A. Miroshnichenko, S. Flach, and Y. Kivshar, Fano resonances in nanoscale structures, Rev. Mod. Phys. 82, 2257 (2010).

[41] M. I. Tribelsky, S. Flach, A. E. Miroshnichenko, A. V. Gorbach, and Y. S. Kivshar, Light Scattering by a Finite Obstacle and Fano Resonances, Phys. Rev. Lett. 100, 043903 (2008). 
[42] Digital Library of Mathematical Functions, http://dlmf. nist.gov/10.49.

[43] M. Garín, R. Fenollosa, P. Ortega, and F. Meseguer, Light harvesting by a spherical silicon microcavity, J. Appl. Phys. 119, 033101 (2016).

[44] M. I. Tribelsky and B. S. Luk'yanchuk, Anomalous Light Scattering by Small Particles, Phys. Rev. Lett. 97, 263902 (2006).
[45] M. Polyanskiy, Refractive Index Database, http:// refractiveindex.info/.

[46] P. Chýlek and J. Zhan, Interference structure of the Mie extinction cross section, J. Opt. Soc. Am. A 6, 1846 (1989).

[47] X. Huang, P. K. Jain, I. H. El-Sayed, and M. A. El-Sayed, Plasmonic photothermal therapy (pptt) using gold nanoparticles, Lasers Med. Sci. 23, 217 (2008). 